\title{
Polymer-Silicate Nanocomposites: Model Systems for Confined Polymers and Polymer Brushes
}

\author{
E. P. Giannelis ${ }^{1,3}$, R. Krishnamoorti ${ }^{2,4}$, E. Manias ${ }^{1,5}$ \\ ${ }^{1}$ Department of Materials Science \& Engineering, Cornell University, Ithaca, NY 14853, USA \\ ${ }^{2}$ Department of Chemical Engineering, University of Houston, Houston, TX 77204, USA \\ 3 emails:epg2@cornell.edu \\ 4 ramanan@bayou.uh.edu \\ 5 manias@msc.cornell.edu
}

The static and dynamic properties of polymer-layered silicate nanocomposites are discussed, in the context of polymers in confined spaces and polymer brushes. A wide range of experimental techniques as applied to these systems are reviewed, and the salient results from these are compared with a mean field thermodynamic model and non-equilibrium molecular dynamics simulations.

Despite the topological constraints imposed by the host lattice, mass transport of the polymer, when entering the galleries defined by adjacent silicate layers, is quite rapid and the polymer chains exhibit mobilities similar to or faster than polymer self-diffusion. However, both the local and global dynamics of the polymer in these nanoscopically confined galleries are dramatically different from those in the bulk. On a local scale, intercalated polymers exhibit simultaneously a fast and a slow mode of relaxation for a wide range of temperatures, with a marked suppression (or even absence) of cooperative dynamics typically associated with the glass transition. On a global scale, relaxation of polymer chains either tethered to or in close proximity $(<1 \mathrm{~nm}$ as in intercalated hybrids) to the host surface are also dramatically altered. In the case of the tethered polymer nanocomposites, similarities are drawn to the dynamics of other intrinsically anisotropic fluids such as ordered block copolymers and smectic liquid crystals. Further, new non-linear viscoelastic phenomena associated with melt-brushes are reported and provide complementary information to those obtained for solution-brushes studied using the Surface Forces Apparatus.

$1 \quad$ Introduction $\ldots \ldots \ldots \ldots \ldots \ldots \ldots \ldots \ldots \ldots \ldots$

$1.1 \quad$ Layered-Silicate Structure . . . . . . . . . . . . . . . 110

2 The Structure of Nanocomposites . . . . . . . . . . . 113

2.1 Morphologies of Polymer/Silicate Nanocomposites . . . . . . . 113

2.2 Thermodynamics of Nanocomposite Formation . . . . . . . . . 115

3 Dynamics of Nanoscopically Confined Polymers . . . . . . . . 118

3.1 Kinetics of Polymer Melt Intercalation . . . . . . . . . . . . . . 118

3.2 Structural Evolution During Intercalation . . . . . . . . . . . . 121

3.3 Local Dynamics . . . . . . . . . . . . . . . . . . . . . . . . 122

3.3.1 On the Relaxation of nm-Thick Polymer Films Between Walls . . 122

3.3.2 NMR Measurements . . . . . . . . . . . . . . . . . . . . . 123

3.3.3 Computer Simulations . . . . . . . . . . . . . . . . . . . 125

3.3.4 Cooperative Motion - TSC and DSC Measuraments . . . . . . . 128 
$4.1 \quad$ Linear Viscoelasticity . . . . . . . . . . . . . . . . . 131

4.2 Alignment of Nanocomposites . . . . . . . . . . . . . . 139

4.3 Strain - Hardening of Polymer Brushes . . . . . . . . . . 140

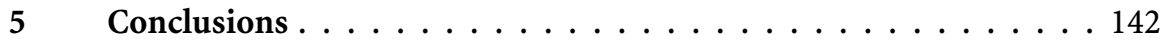

6 References. . . . . . . . . . . . . . . . . . . . 143

\section{Introduction}

Layered-silicate based polymer nanocomposites have become an attractive set of organic-inorganic materials not only for their obvious potential as technological materials, but also for providing a convenient macroscopic system to study fundamental scientific issues concerning confined and tethered polymers. Studying the formation, structure and dynamics of these nanocomposites can lead to a better understanding of organic-inorganic hybrids, polymers in a confined environment or at a solid interface and polymer brushes.

One promising way to synthesize polymer nanocomposites is by intercalating polymers in layered inorganic hosts [1-4]. Graphite, transition metal chalcogenides, metal phosphates, complex oxides, oxychlorides and mica-type layered silicates are some examples of layered solids capable of intercalation. The structure and properties of the resulting nanostructure can be conveniently mediated by controlling subtle guest-host interactions. Beyond the conventional phase separated polymer/silicate composites, for which the polymer and the inorganic host remain immiscible, two types of hybrids are possible (Fig. 1): intercalated in which a single, extended polymer chain is intercalated between the host layers resulting in a well ordered multilayer with alternating polymer/inorganic layers and a repeat distance of a few nanometers (Fig. 2a), and exfoliated or delaminated, in which the silicate layers ( $1 \mathrm{~nm}$ thick) are exfoliated and dispersed in a continuous polymer matrix $[5,6]$ (Fig. $2 b$ ).

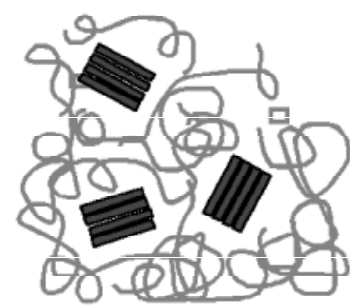

Phase separated

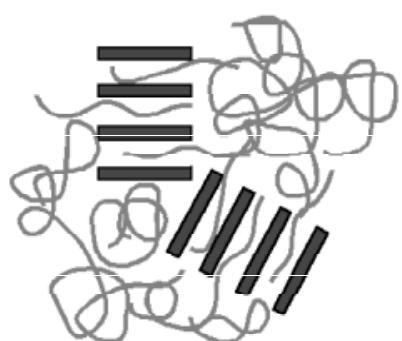

Intercalated

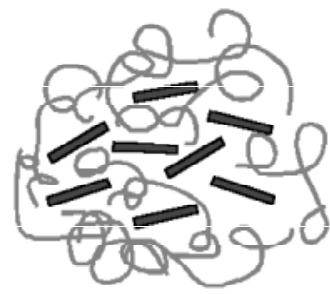

Exfoliated

Fig. 1. Schematic representation of different polymer/silicate hybrid structures. 


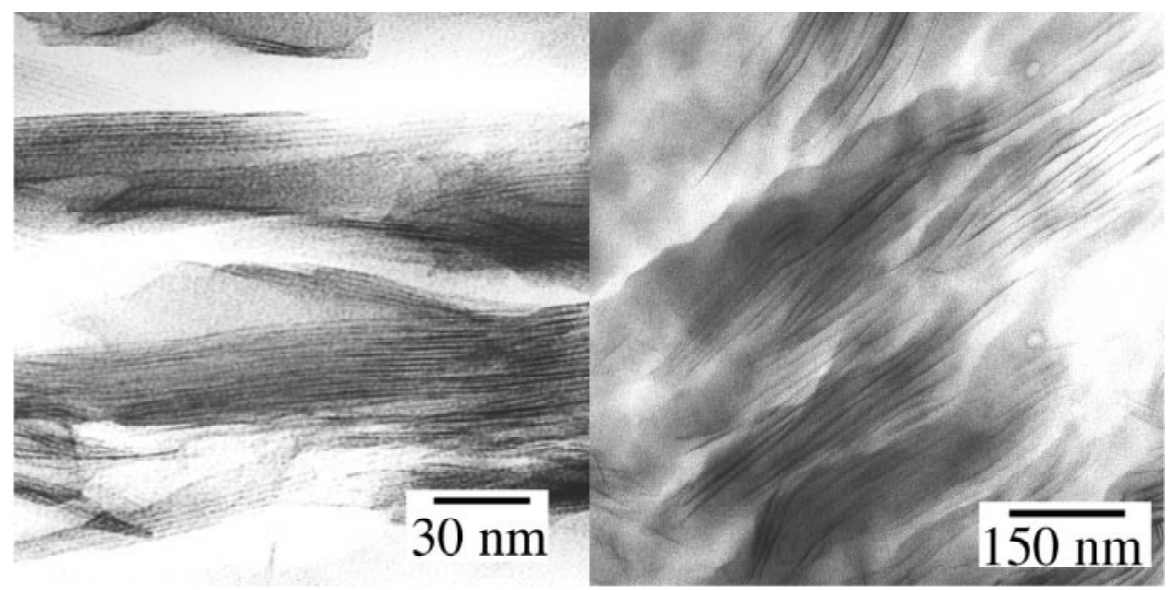

Fig. 2. TEM images of an intercalated (left) and an exfoliated (right) hybrid [5]

In the case of mica-type layered silicates it has been recently demonstrated that nanocomposites (both intercalated and delaminated) can be synthesized by direct melt intercalation even with high molecular weight polymers [7-18]. This synthetic method is quite general and is broadly applicable to a range of commodity polymers from essentially non-polar polystyrene, to weakly polar poly(ethylene terephthalate), to strongly polar nylon. Nanocomposites can, therefore, be processed using currently available techniques such as extrusion, thus lowering the barrier towards commercialization.

The unprecedented mechanical properties of polymer layered silicate (PLS) nanocomposites were first demonstrated by a group at the Toyota research center in Japan using nylon nanocomposites [19-21]. They showed that a doubling of the tensile modulus and strength is achieved for nylon-layered silicate nanocomposites containing as little as 2 vol. \% inorganic. More importantly the heat distortion temperature of the nanocomposites increases by up to $100^{\circ} \mathrm{C}$ extending the use of the composite to higher temperature environments, such as automotive under-the-hood parts.

PLS nanocomposites have several advantages [6] including: (a) they are lighter in weight compared to conventionally filled polymers because high degrees of stiffness and strength are realized with far less high density inorganic material; (b) their mechanical properties are potentially superior to fiber reinforced polymers because reinforcement from the inorganic layers will occur in two rather than in one dimension without special efforts to laminate the composites; and (c) they exhibit outstanding diffusional barrier properties without requiring a multipolymer layered design, allowing for recycling.

In addition to their potential applications, PLS nanocomposites are unique model systems to study the statics and dynamics of polymers in confined environments. Using both delaminated and intercalated hybrids, the statics and dy- 
namics of polymers confined over distances ranging from the radius of gyration of the polymer to the statistical segment length of the chains can be studied. Even simple notions regarding the conformations of polymers confined to two dimensions are yet to be understood. In three dimensions it is known that for long chain polymers there is significant overlap between molecules. In two-dimensions it has been suggested that different chains should only overlap slightly [22]. Therefore the local and global conformations of the polymers within the host galleries are expected to be dramatically different from those observed in the bulk not only due to the confinement of the polymer chains but also due to specific polymer-surface interactions, normally not observed in the bulk. It is also expected that the local and chain dynamics would be greatly affected by the confinement as well as the polymer-surface interactions.

The behavior of polymer liquids under confinement is in general very different from that in the bulk, especially when the confining dimensions become comparable or smaller than the polymer coil size. Traditional notions such as reptation dynamics governing the relaxation of a long polymer chain are improbable in highly confined intercalated systems (confinement distances comparable to the statistical segment length of the polymer) as it is impossible to imagine a topological entanglement in two dimensions.

\section{1}

\section{Layered-Silicate Structure}

The layered silicates used in the nanocomposites belong to the same structural family as the better known minerals talc and mica [10] (i.e. 2:1 phyllosilicates). Their crystal lattice consists of a two-dimensional, $1 \mathrm{~nm}$ thick layers which are made up of two tetrahedral sheets of silica fused to an edge-shaped octahedral sheet of alumina or magnesia. The lateral dimensions of these layers vary from $300 \AA$ to several microns depending on the particular silicate. Stacking of the layers leads to a regular van der Walls gap between them called the interlayer or gallery. Isomorphic substitution within the layer generates negative charges that are normally counterbalanced by hydrated alkali or alkaline earth cations residing in the interlayer. Because of the relatively weak forces between the layers (due to the layered structure), intercalation of various molecules, even polymers, between the layers is facile.

Pristine mica-type layered silicates usually contain hydrated $\mathrm{Na}^{+}$or $\mathrm{K}^{+}$ions [16]. Ion exchange reactions with cationic surfactants including primary, tertiary and quaternary ammonium or phosphonium ions render the normally hydrophilic silicate surface organophilic, which makes possible intercalation of many engineering polymers. The role of alkyl ammonium cations in the organosilicates is to lower the surface energy of the inorganic host and improve the wetting characteristics with the polymer. Additionally, the alkyl ammonium cations could provide functional groups that can react with the polymer or initiate polymerization of monomers to improve the strength of the interface between the inorganic and the polymer [17-19]. 


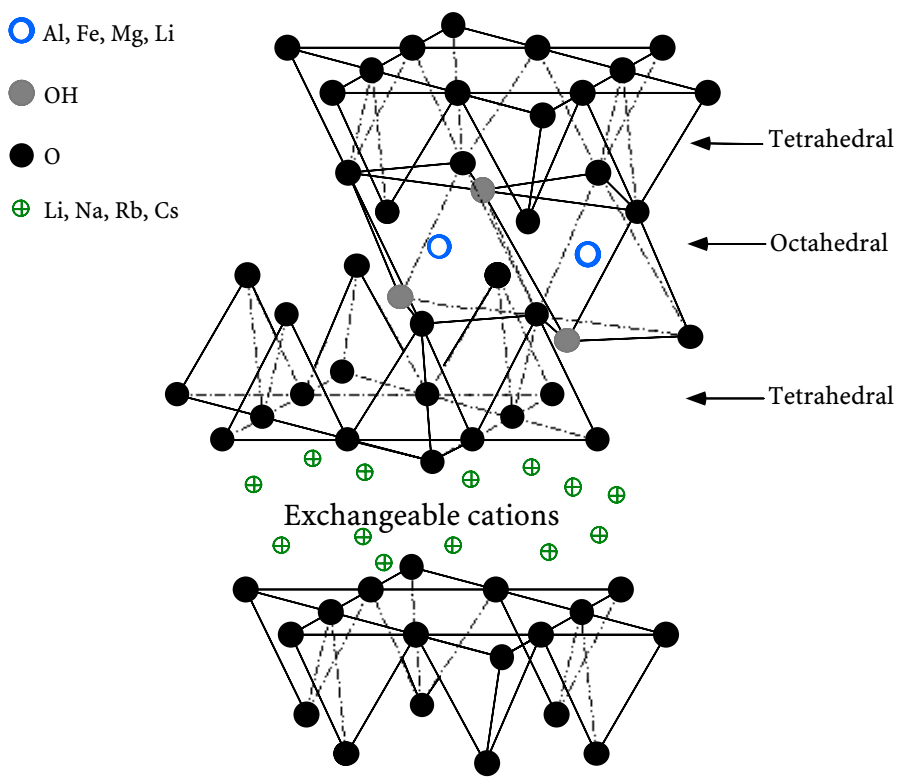

Schematic 1. The structure of 2:1 layered silicates. $M$ is a monovalent charge compensating cation in the interlayer and $\mathrm{x}$ is thedegree of isomorphous substitution, which for the silicates of interest is between 0.5 and 1.3. The degree of isomorphous substitution is also expressed as a cation exchange capacity (CEC) and is measured in milli-equivalents/g.

The most commonly used layered silicates are montmorillonite, hectorite and saponite. Details on the structure and chemistry for these layered silicates are provided in Schematic 1 and Table 1 . All of these silicates are characterized by a large active surface area $\left(700-800 \mathrm{~m}^{2} / \mathrm{g}\right.$ in the case of montmorillonite), a moderate negative surface charge (cation exchange capacity) (CEC) and layer morphology, and are regarded as hydrophobic colloids of the constant-charge type. The layer charge indicated by the chemical formula is only to be regarded as an average over the whole crystal because the charge varies from layer to layer (within certain bounds). Only a small proportion of the charge balancing cations are located at

Table 1. Structure and chemistry of Mica-type layered silicates

\begin{tabular}{|c|c|c|}
\hline Silicate & $\begin{array}{l}\text { Location of } \\
\text { isomorphous } \\
\text { substitution }\end{array}$ & Formula \\
\hline $\begin{array}{l}\text { Montmorillonite } \\
\text { Hectorite } \\
\text { Saponite }\end{array}$ & $\begin{array}{l}\text { Octahedral } \\
\text { Octahedral } \\
\text { Tetrahedral }\end{array}$ & $\begin{array}{l}\mathrm{M}_{\mathrm{x}}\left[\mathrm{Al}_{4-\mathrm{x}} \mathrm{Mg}_{\mathrm{x}}\right]\left(\mathrm{Si}_{8}\right) \mathrm{O}_{20}(\mathrm{OH})_{4} \\
\mathrm{M}_{\mathrm{x}}\left[\mathrm{Mg}_{6-\mathrm{x}} \mathrm{Li}_{\mathrm{x}}\right]\left(\mathrm{Si}_{8}\right) \mathrm{O}_{20}(\mathrm{OH})_{4} \\
\mathrm{M}_{\mathrm{x}}\left[\mathrm{Mg}_{6}\right]\left(\mathrm{Si}_{8-\mathrm{x}} \mathrm{Al}_{\mathrm{x}}\right) \mathrm{O}_{20}(\mathrm{OH})_{4}\end{array}$ \\
\hline
\end{tabular}



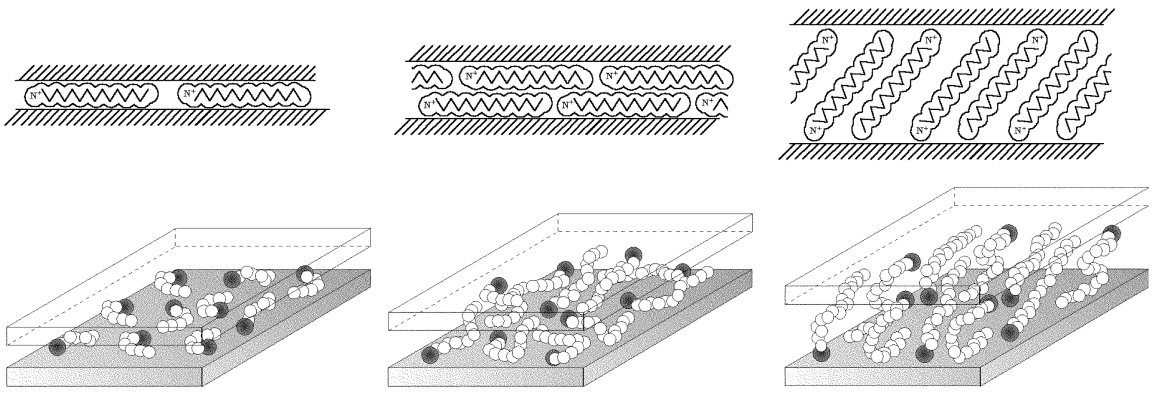

Fig. 3. (top) Idealized structures of organically modified silicates; adopted from Weiss A. (1963) Angew Chem 2: 134 and Lagaly G (1976) Angew Chem 15: 575. (bottom) The structure of organically modified silicates as derived from FTIR experiments (adopted from Vaia et al. (1994) Chem Mater 6: 1017) and molecular dynamics computer simulations of Hackett et al. (1998) J. Chem. Phys. 108: 7410.
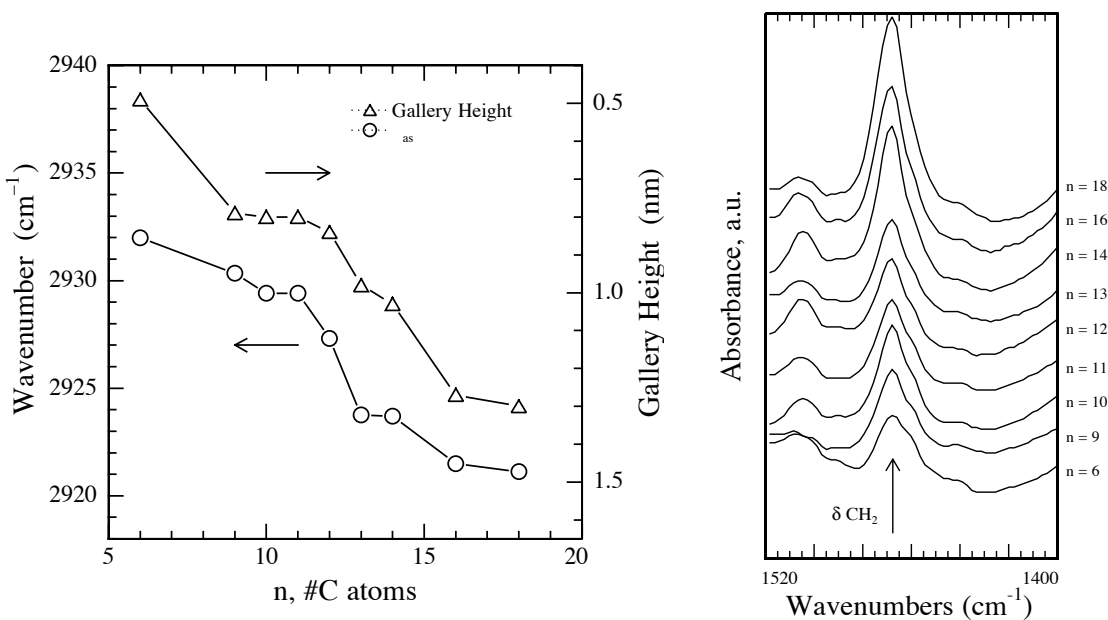

Fig. 4. FTIR studies of organically modified silicates. (left) Asymmetric $\mathrm{CH}_{2}$ stretch $\left(\nu_{\mathrm{as}}\left(\mathrm{CH}_{2}\right)\right)$ and gallery height as a function of surfactant chain length measured at room temperature for a series of organically modified fluorohectorites. The large variation in $\nu_{\mathrm{as}}$ and gallery height suggests a wide range of molecular configurations and not an all-trans surfactant arrangement [9]. (right) Variation in the $\mathrm{CH}_{2}$ bending (scissoring) vibration, $\delta\left(\mathrm{CH}_{2}\right)$, for a series of organically modified fluorohectorites at room temperature. The observed absoption at $1468 \mathrm{~cm}^{-1}$ is characteristic of a partially ordered phase where the chains are mobile while maintaining some orientational order [9].

the external crystal surface with the majority being present in the interlayer space. The cations are exchangeable for others in solution.

Upon replacing the hydrated metal cation from the interlayers in the pristine layered silicates with organic cations such as an alkylammonium or an alkylphosphonium, the layered silicate attains a hydrophobic/organophillic character 
and typically results in a larger interlayer spacing. Because the negative charge originates in the silicate layer, the cationic head group of the alkylammonium molecule preferentially resides at the layer surface and the aliphatic tail will radiate away from the surface. The equilibrium layer spacing for an organically modified layered silicate depends both on the cation exchange capacity of the layered silicate, as well as on the chain length of the organic cation. Traditional structural characterization to determine the orientation and arrangement of the alkyl chain involved primarily the use of X-ray diffraction. Depending on the packing density, temperature and chain length, the chains were thought to lie either parallel to the silicate layers forming mono or bilayers, or radiate away from the surface forming mono or bimolecular tilted arrangements (Fig. 3). These idealized structures have been shown to be not-realistic by Vaia and coworkers using FTIR experiments [9]. They have shown that the alkyl chains can vary from liquid-like to solid-like, with the liquid-like structure dominating as the interlayer density or chain length decreases (Fig. 4), or as the temperature increases. This can be understood because of the relatively small energy differences between the trans and gauche conformers; the idealized models described earlier assume all trans conformations. In addition, for the longer chain length surfactants, the surfactants in the layered silicate can show thermal transitions akin to melting or liquid-crystalline to liquidlike transitions upon heating.

\section{2}

\section{The Structure of Nanocomposites}

\section{1}

\section{Morphologies of Polymer/Silicate Nanocomposites}

The structure of the PLS nanocomposites has traditionally been elucidated using X-ray diffraction (XRD) and transmission electron microscopy (TEM) [1,2]. Due to the periodic arrangement of the silicate layers both in the pristine and the intercalated states, with periodicity of 1-4 $\mathrm{nm}$ and the presence of high atomic number species in the layers, the choice of X-ray diffraction in determining the interlayer spacing is obvious. However, in the absence of registry, as in an exfoliated or a delaminated nanocomposite, as well as in a disordered nanocomposite, XRD does not provide definite information regarding the structure of the nanocomposite (Fig. 5). In order to provide quantitative information in XRD 'silent' nanocomposites, TEM has proven to be an extremely useful technique. In addition to a description of the spatial correlations of the layered silicates, TEM also provides a means to discern the homogeneity of the mixing process. A bright field TEM image of an organically modified layered silicate intercalated with polystyrene is shown in Fig. 2a. The periodic alternating dark and light bands represent the layers of silicate and the interlayers respectively, with a spacing of $\sim 3 \mathrm{~nm}$ between the silicate layers. The TEM also reveals the presence of individual crystallites consisting of several tens of such silicate layers, with bulk polymer filling the space between crystallites. The pristine organically modified 


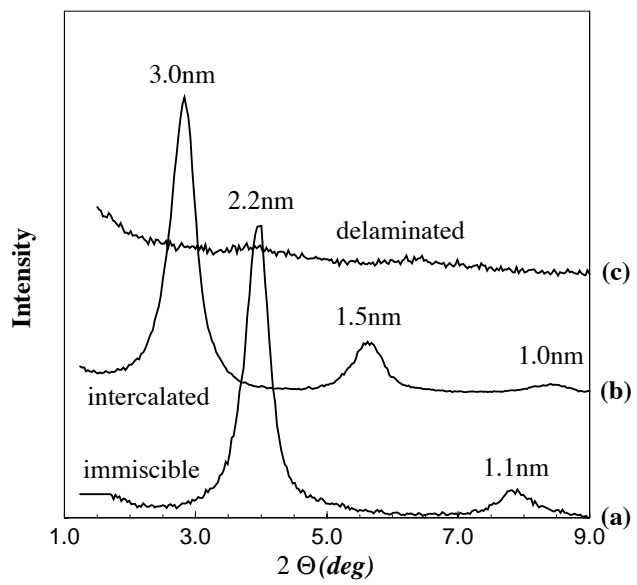

Fig. 5. Typical XRD patterns from polymer/silicate hybrids. (a) XRD obtained from an "immiscible" system (here polyethylene/C18FH), and is identical with the XRD of the neat organo-silicate $(\mathrm{C} 18 \mathrm{FH})$. For intercalated hybrids the d-spacing shifts to a higher value (b) as the gallery expands to accommodate the intercalating polymer (here polystyrene/ C18FH); second and third order reflections - as shown here - are very common and some times intercalated hybrids can have up to 13 order reflections [7], manifesting a remarkable longrange registry. (c) Typical XRD of an exfoliated/delaminated structure or a disordered system (here a siloxane/C18FH delaminated hybrid).

layered silicate exhibits the same microstructure as that observed in Fig. 2a, with the exception of a smaller interlayer spacing. A TEM of an exfoliated hybrid of an organically modified layered silicate dispersed in epoxy is shown in Fig. 2b. Such a hybrid, while being XRD silent, does in fact exhibit some relative layer order with spacing of a few tens of nm between layers. This is attributed to the intrinsic anisotropic dimensions of the layers with the aspect ratios of the individual layers being in the range of 100-1000. In addition, flexibility of the nanometer thick silicate layers is evident in this micrograph. Similar micrographs have also been obtained by Lan and coworkers [4] and the Toyota group using monomer intercalation followed by polymerization. Micrographs along with small angle X-ray scattering studies of oriented nanocomposites by the Toyota group [19-21] clearly demonstrate the difference observed in short length scale correlations in unaligned exfoliated nanocomposites (as present in Fig. 2b) and the long range correlations that are observed in aligned (using external shear and elongational flows) nanocomposites. These features will be discussed later (see Section 4.2).

Recently, several attempts have been made to understand the underlying structural aspects of the silicate layers as well as the conformations of polymers in layered silicate based nanocomposites, using techniques such as small angle neutron scattering (SANS) and dynamic light scattering. Jinnai and coworkers [23] have studied mixtures of an organically modified vermiculite (modified with n-butylammonium) with poly(vinyl methyl ether) in the presence of n-butylam- 
monium chloride and heavy water, using neutron scattering in order to elucidate the degree of chain interpenetration trapped in lamellar systems. They concluded that the introduction of polymer causes the silicate layers to become more strongly aligned with more regular, but generally decreasing, interlayer spacing in the gel phase. However, the addition of polymer had no effect on the phase transition temperature between the tactoid and gel phases of the layered silicate.

However, the conformation and location of the polymer chains in these mixtures were not unequivocally determined. Carrado et al. [24] have recently used SANS to monitor the change in the structure of the layered silicate (synthetic hectorites) upon hydrothermal crystallization with direct incorporation of poly(vinyl alcohol) (PVA). The results of these experiments established that the PVA appears to coat the small initially formed silicate particles, hindering their further growth. However, upon removal of the polymer no change has been observed in the extended inorganic network. Muzny and coworkers [25] have applied dynamic light scattering to monitor the dispersion of layered silicates in a polymer matrix. Specifically the dispersion of synthetic hectorite clay platelets suitably organically modified in a matrix of polyacrylamide was studied. These studies have clearly shown that a homogeneous ('single layer dispersion') was achievable only when a large excess (equivalent to five times the CEC of the silicate or higher) of the organic cationic surfactant was used.

\section{2}

\section{Thermodynamics of Nanocomposite Formation}

The formation and equilibrium structure of polymer layered silicate nanocomposites, in particular with organically modified layered silicates, has been shown to be a strong function of the nature of the polymer (polar or apolar), the charge carrying capacity of the layered silicate, as well as the chain length and structure of the cationic surfactant. However, both the polymer/silicate compatibility and hybrid equilibrium structure for these nanocomposites are observed to be independent of polymer molecular weight. The experimental results have been summarized by Vaia et al. and a lattice based mean field theory has been developed to explain these results [26].

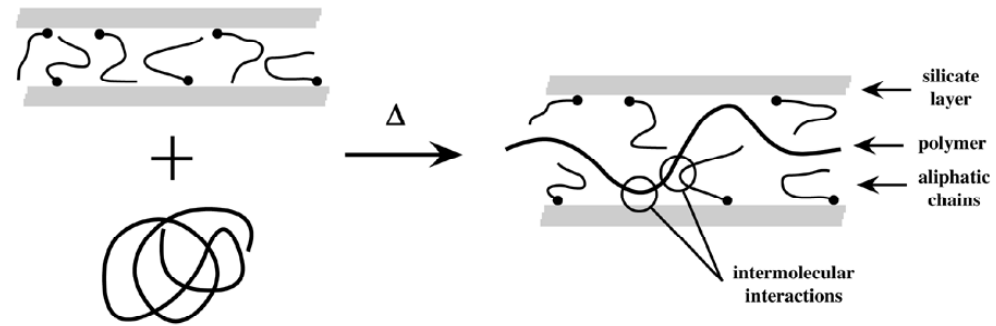

Schematic 2. Schematic representation of the system components before and after the intercalation takes place. The changes in entropy and free energy as a function of the change in gallery height are shown in Fig. 6 and 7. 
In general, an interplay of entropic and enthalpic factors determines the outcome of polymer intercalation. Confinement of the polymer inside the interlayers results in a decrease in the overall entropy of the polymer chains. However, the entropic penalty of polymer confinement may be compensated by the increased conformational freedom of the tethered surfactant chains in a less confined environment, as the layers separate (Fig. 6). Since for small increases in gallery height the total entropy change is small, modest changes in the system's total enthalpy will determine if intercalation is thermodynamically possible. Complete layer separation, though, depends on the establishment of very favorable polymer-surface interactions to overcome the penalty of polymer confinement (Fig. 7). The enthalpy of mixing can broadly be classified into two components - apolar, which is generally unfavorable, and polar which originates from the Lewis acid/Lewis base character of the layered silicates, and which could be rendered favorable. A favorable enthalpy change is accentuated by maximizing the magnitude and number of favorable polymer-surface interactions while minimizing the magnitude and number of unfavorable apolar interactions between the polymer and the functionalizing aliphatic chains.

Although simple in comparison to contemporary thermodynamic descriptions of polymer phenomena, the greatest advantage of the current model is the ability to analytically determine the effect of various aspects of the polymer and OLS on hybrid formation. The variation of the free energy of mixing on the gallery spacing and its dependence on enthalpic and entropic factors, based on this model, suggest three possible equilibrium states - immiscible, intercalated and exfoliated. The model has been successful in addressing some of the fundamen-

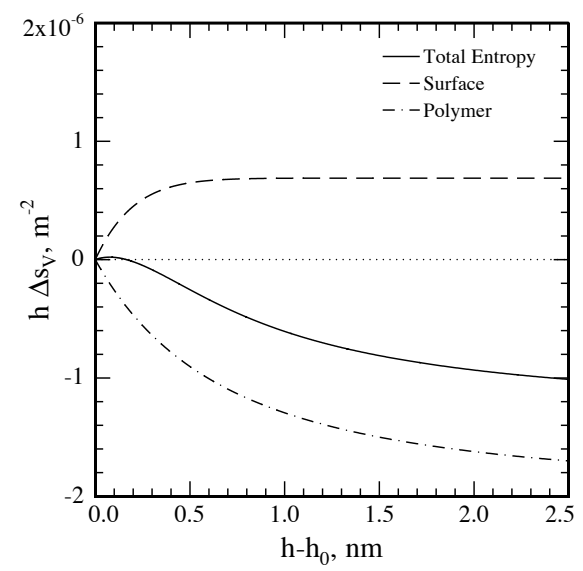

Fig. 6. The change of entropy per area versus the change in gallery height, for the polymer and the surfactant (octadecylammonium) functionalized surface based on the thermodynamic model presented in [26]. 


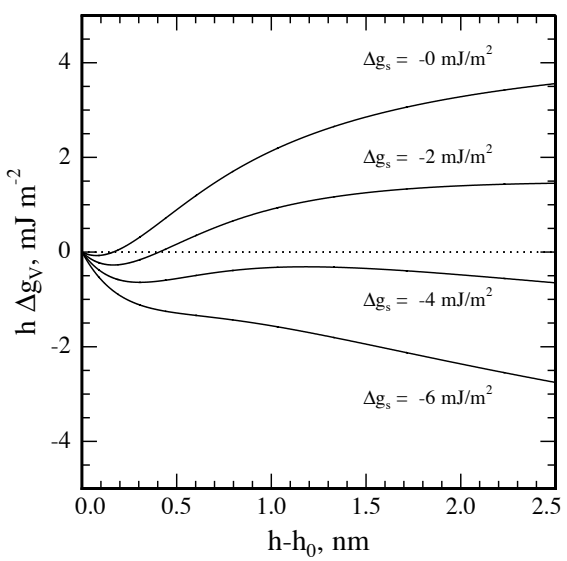

Fig. 7. The change of free energy per area versus the change in gallery height based on the thermodynamic model presented in [26], for various surface-polymer affinities: $\Delta g_{s}=0,-2$, -4 and $-6 \mathrm{~mJ} / \mathrm{m}^{2}$. Both figures adopted from [26]

tal and qualitative thermodynamic issues associated with hybrid formation. However, assumptions such as the separation of configurational terms and intermolecular interactions and the further separation of the entropic behavior of the constituents, somewhat limit the usefulness of the model. Additionally, this model is not applicable to situations where the OLS's interlayer is not completely occupied by tethered-chain segments, which is the case for many silicates with low charge densities or modified by short aliphatic chains.

Some of the limitations and gross approximations of the current mean-field, lattice-based model, such as the decoupling of the tethered chain and polymer conformations in the interlayer, may be addressed by developing more sophisticated models of hybrid formation based on theories of Scheutjens and Fleer [27], and Szleifer [28]. Additionally, Monte Carlo simulations of hybrid formation or intercalation of small-molecules in layered materials could lead to additional insights into the mechanisms and important factors associated with intercalation [14]. One very well studied problem is the hydration (water intercalation) in 2:1 silicates which was approached both by Monte Carlo [29, a-c] and molecular dynamic simulations [29, d]. In Fig. 8 the water uptake as a function of gallery height as provided by Grand Canonical MC simulations is shown. From the simulation the thermodynamic properties of the systems - such as disjoining pressure and free energy - can be obtained, and thus the stable d-spacings can be located. The extension of such simulation schemes to the study of polymer/organo-silicate systems is currently underway, and will obviously provide a more detailed picture of the thermodynamics of these systems [29e]. 

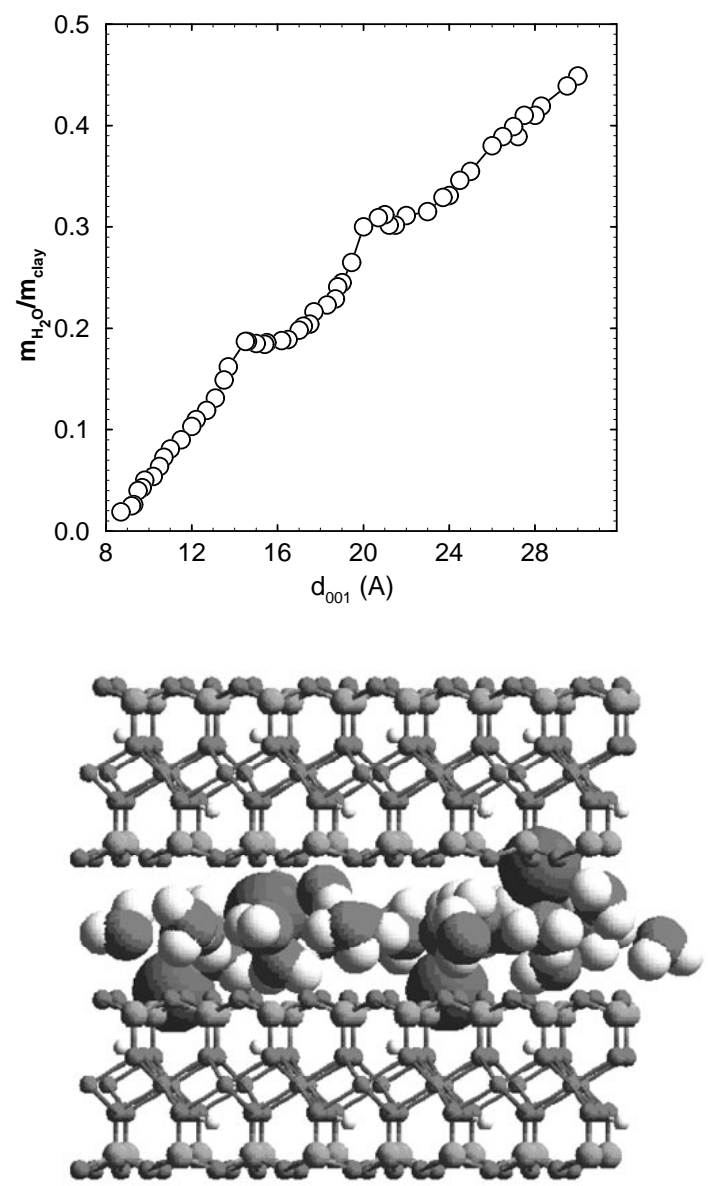

Fig. 8. Simulated water uptake from Monte Carlo simulations, adopted from [29 c]. A typical configuration of a hydrated $\mathrm{Na}^{+}$montmorillonite in the $\mathrm{d}_{001}=12 \AA$ stable hydrated state revealed by Monte Carlo simulations [29].

\section{3}

\section{Dynamics of Nanoscopically Confined Polymers}

\section{1}

\section{Kinetics of Polymer Melt Intercalation}

As outlined in the introduction, polymer melts can intercalate layered inorganic compounds unassisted by shear or solvents. This is a rather surprising result as it implies that polymer chains can undergo large center of mass displacement in almost two dimensional interstices as the distances between the confining sur- 
faces are substantially smaller than the unperturbed radius of gyration of the polymer and are comparable to the monomer size. The reduction in free energy by the intercalate formation (Section 2.2) and the concentration gradient during the intercalation process, give rise to an 'enthalpic force' which 'drives' the polymer coils into the interlayer galleries. On the other hand, the conformational energy cost of stretching the chains, in addition to the topographical constrains and the adsorption on the surfaces are expected to impose severe limitations on diffusion of chains diffusing in a pseudo-two dimensional slit.

Vaia et al. [12] have observed that the kinetics of intercalation even under quiescent conditions (absence of external shear) are quite rapid. Using in-situ XRD (which monitors the angular shift and integrated intensity of the silicate reflections, Fig. 9) they studied the intercalation kinetics of model polymers (monodisperse polystyrene) in organically modified fluorohectorite.

Figure 9 shows a typical temporal series XRD patterns, for a polystyrene $M_{w}=$ 30,000 (PS30)/octadecyl-ammonium modified fluorohectorite (C18FH) mixture annealed in-situ at $160^{\circ} \mathrm{C}$ in vacuum. Details regarding the data collection and analysis are presented in reference [12]. The width of the original unintercalated peak and the final intercalated peak appear to be similar, suggesting that the polystyrene melt intercalation does not drastically alter the coherence length or disrupt the layer structure of the silicate crystallites.

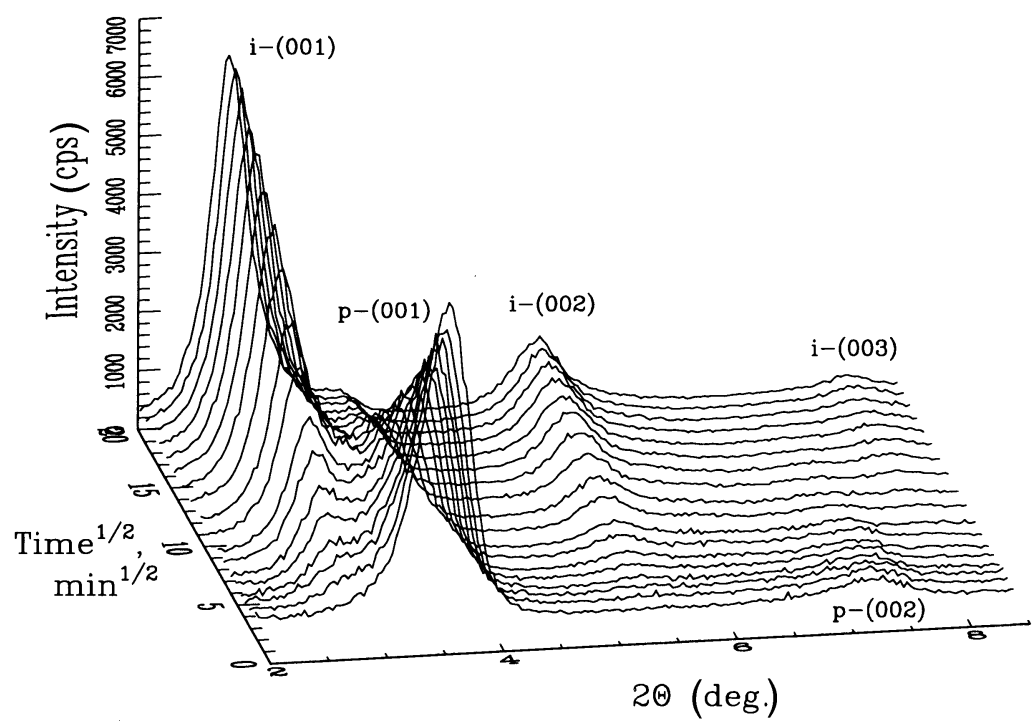

Fig. 9. Typical temporal series of XRD patterns, for a polystyrene $\left(M_{w}=30,000\right) / C 18 \mathrm{FH}$ mixture annealed in-situ at $160^{\circ} \mathrm{C}$ in vacuum. Initially (time $=0$ ), the basal reflection of the organo-silicate were observed at $2 \theta=4.15^{\circ}(\mathrm{d}=2.13 \mathrm{~nm})$. During the annealing this reflection is progressively reduced in intensity, whereas a new basal reflection develops at $2 \theta=2.82^{\circ}$ $(\mathrm{d}=3.13 \mathrm{~nm})$ that corresponds to the intercalated hybrid. 
Moreover the evolution of the XRD during annealing can be modeled to determine the apparent diffusivity, $\mathrm{D}$, of the polymers within the silicate gallery. Namely, the ratio of the amount of intercalated polymer at time $t, Q(t)$, to that at equilibrium $Q(\infty)$ is [30]:

$$
\frac{Q(t)}{Q(\infty)}=1-\sum_{m=1}^{\infty} \frac{4}{a_{m}^{2}} \exp \left(-\frac{D}{\bar{a}^{2}} a_{m} t\right)
$$

where, $D / \bar{a}^{2}$ is the effective diffusional coefficient, $\bar{a}$ is the mean size of the impermeable surface and $a_{m}$ are the roots of the zeroth order Bessel function $\left(J_{0}(a)=0\right)$. The fraction of intercalated polymer is given by the corresponding fraction of the XRD integrated intensities $\chi$ (Fig. 10) and the only adjustable parameter is $D / \bar{a}^{2}$; the mean silicate size $\bar{a}$ can be measured directly by TEM [12] and in that case was approximately $5 \mu \mathrm{m}$.

The apparent diffusivity for the intercalation of PS30 in C18FH is the same order of magnitude $\left(10^{-11} \mathrm{~cm}^{2} / \mathrm{s}\right.$ at $\left.170^{\circ} \mathrm{C}\right)[12]$ as the self diffusion coefficient of polystyrene determined at comparable temperatures and molecular weights [31]. Furthermore, the activation energy of melt intercalation is $166 \pm 12 \mathrm{~kJ} / \mathrm{mol}$ which is comparable to the activation energy measured for self diffusion of polystyrene $(167 \mathrm{~kJ} / \mathrm{mol}$ [31]). Although these first results seem to suggest that the process of intercalation is dictated by the transport of polymer to the silicate agglomerates and not by any process by which the polymer is moved inside the galleries of the silicates [12], more recent investigations - where the effect of the polymer-surface interactions on the intercalation kinetics were explored [32] - have proved that
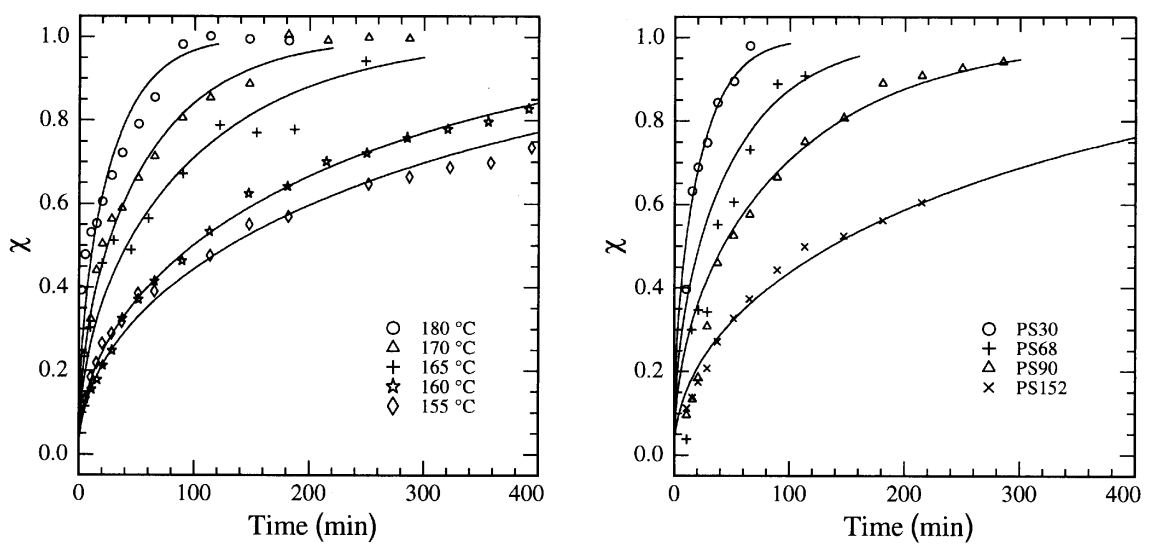

Fig. 10. The fraction of polystyrene intercalated $\mathrm{C} 18 \mathrm{FH}$ for various annealing temperatures (PS 30, $\mathrm{M}_{\mathrm{w}}=30,000$ ) (left) and for various polymer molecular weights at $180^{\circ} \mathrm{C}$ (right). The lines are the best fits to the data using equation 1.1. The molecular weights of polystyrene used were $\mathrm{M}_{\mathrm{w}}=30,000$ (PS30), $\mathrm{M}_{\mathrm{w}}=68,000$ (PS68) $\mathrm{M}_{\mathrm{w}}=90,000$ (PS90) and $\mathrm{M}_{\mathrm{w}}=152,000$ (PS152); adopted from reference [12]. 
this is not always the case. In these more recent studies, it was found that the effective diffusion coefficient depends markedly on the surfactant used, for the same polymer and annealing temperature. Because the surfactant can only affect the polymer motion inside the galleries, one concludes that this motion of the intercalated polymer is the process which dictates the intercalation kinetics [33]. Furthermore, for some systems the intercalating polymers were found to possess a mobility that was much faster than the self-diffusion coefficient of the corresponding polymer in the bulk [31], or in a thin film [34]. This is not unexpected since intercalation is a process where polymers are moving down a concentration gradient, whereas in the other two cases the polymer motion is entropic in origin [35].

\section{2}

\section{Structural Evolution During Intercalation}

The microstructural evolution during the polymer melt intercalation process (leading to well ordered intercalated compounds, disordered intercalated composites or exfoliated nanocomposites) has been studied recently using a combination of XRD, conventional TEM, and high resolution TEM. For well ordered intercalates, as shown in Fig. 2a, the microstructure appears to be very similar to that of the unintercalated layered silicates. The individual crystallites of the layered silicate are tightly packed and oriented along the major axis of the primary particle, with slits between crystallites of comparable dimensions to the gallery height between the silicate layers within a crystallite (Fig. 11). Micrographs ob-

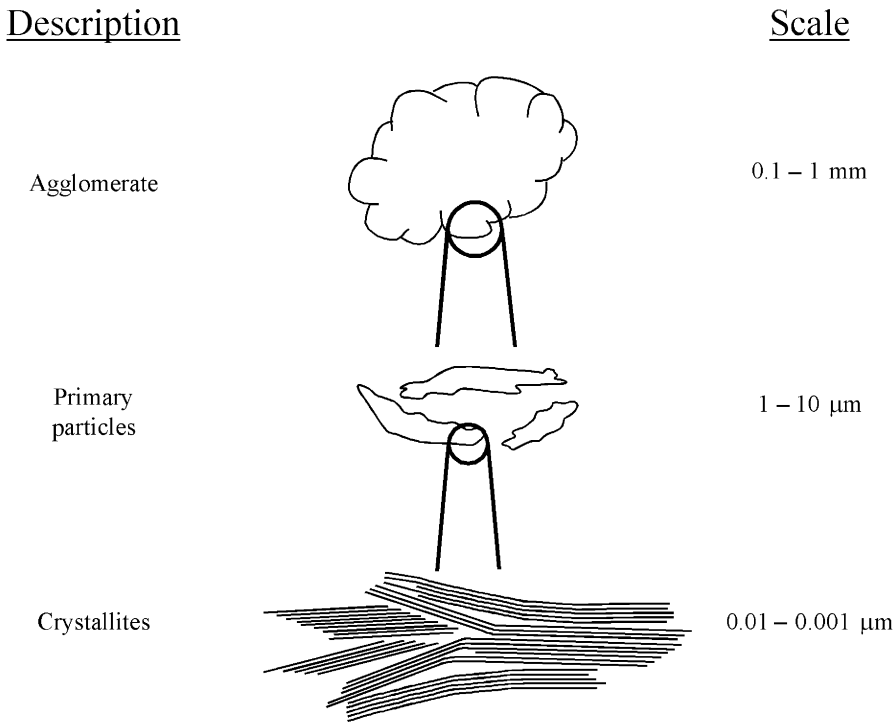

Fig. 11. Hierarchy of structures in a silicate, from reference [12]. 
tained on partially intercalated nanocomposites revealed the presence of unintercalated crystallites towards the interior of the primary particle (away from the primary particle - bulk polymer interface, where the intercalated crystallites are found), with no crystallites containing both intercalated and unintercalated layers. Based on these observations, it was concluded that polymer intercalation occurs as a front which penetrates the primary silicate particle from the exterior.

In the case of disordered intercalated and exfoliated nanocomposites (examples being polystyrene with a dodecyl-ammonium modified fluorohectorite $(\mathrm{C} 12 \mathrm{FH})$ and poly(3-bromostyrene) (PS3Br) with a C12FH respectively), XRD does not produce well defined basal reflections, and in fact, produces a featureless pattern for the exfoliated nanocomposite. TEM of the PS/C12FH nanocomposite revealed the microstructure of the primary silicate particle to be heterogeneous, with regions of coherent order more prevalent toward the interior than near the bulk polymer - primary particle interface. Layer spacings were found to range from 2.1 to $6.0 \mathrm{~nm}$, with a mean layer spacing of $\sim 2.8 \mathrm{~nm}$, comparable to that obtained from the diffuse peak observed in the XRD. In the case of the PS3Br/C12FH nanocomposites, the micrographs indicated that while individual layers were found dispersed in the matrix of the polymer, due to the extremely large aspect ratios of the layers, preferential alignment of the layers even at large separations $(>10 \mathrm{~nm})$ was observed. Extensive polymer penetration of the interlayer galleries was observed along with enhanced inter-crystallite gaps within the primary particle. While the layers appear well separated in both the interior and boundaries of the primary particle, the relative order between layers is much better preserved in the interior portions, suggesting that the delamination process occurs by different mechanisms near the bulk polymer - primary particle boundaries, as opposed to the interior of the interlayers.

\section{3}

\section{Local Dynamics}

\subsection{1}

\section{On the Relaxation of nm-Thick Polymer Films Between Walls}

Intercalated compounds offer a unique avenue for studying the static and dynamic properties of small molecules and macromolecules in a confined environment. More specifically, layered nanocomposites are ideal model systems to study small molecule and polymer dynamics in restrictive environments with conventional analytical techniques, such as thermal analysis, NMR, dielectric spectroscopy and inelastic neutron scattering. Understanding the changes in the dynamics due to this extreme confinement (layer spacing $\ll \mathrm{R}_{\mathrm{g}}$ and comparable to the statistical segment length of the polymer) would provide complementary information to those obtained from traditional Surface-Force Apparatus (SFA) measurements on confined polymers (confinement distances comparable to $\mathrm{R}_{g}$ ) [36]. 


\subsection{2}

\section{NMR Measurements}

The local dynamics of segments of polymer chains can be very conveniently probed using solid-state NMR. The local dynamics of intercalated chains of polyethylene oxide (PEO) in a pristine layered silicate were studied using variable temperature solid state NMR [13]. Figure 13 compares the ${ }^{2} \mathrm{H}$ line shapes for bulk d-PEO and intercalated d-PEO (in Li-fluorohectorite), wherein the d-PEO chains $\left(M_{w}=180,000, M_{w} / M_{n}=1.2\right.$, Polysciences) are confined to a $0.8 \mathrm{~nm}$ gap between the silicate layers. For the bulk polymer, at the lowest temperatures where the local segmental motion of the polymer is quiescent, the NMR signal possesses well-formed singularities and sharp step-edges characteristic of the solid/glassy dynamics. With increasing temperature the 'sharp' spectral features gradually broaden with a build-up of the intensity at the center of the powder pattern, indicative of the onset of polymer dynamics. The central peak results from increased segmental motion which causes temporal averaging of the signal. At the highest temperatures i.e., in the melt state of the polymer, where the dynamics of the polymer is expected to be fairly rapid, the anisotropic pattern disappears and a single line spectrum is obtained. In contrast, the intercalated polymer chains even at the lowest experimental temperature of $220 \mathrm{~K}$ exhibit small amplitude dynamics as evidenced by the loss of spectral definition. With increasing temperature the powder pattern shows some narrowing and a progressive increase in intensity at the center, consistent with progressively increasing rates of C-D bond re-orientations. However, unlike the bulk sample, the intercalated sample at $400 \mathrm{~K}$ (well above any bulk dynamic transition) displays significant spectral features other than the central peak, indicative of significant preferential orientation of the trapped polymer segments with respect to the silicate structure confining the polymer. The temperature dependence of the line shapes indicates that the intercalated chains have more 'freedom' to sample a

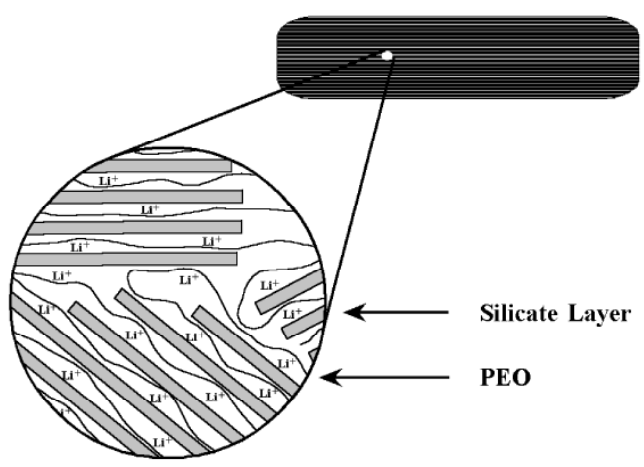

Fig. 12. Schematic representation of the $\mathrm{PEO} / \mathrm{Li}^{+}$fluorohectorite intercalated hybrid. 
distribution of local chain configurations, resulting in increased signal averaging. However, at the highest temperatures, the intercalated d-PEO still exhibits a broad base structure reminiscent of the powder pattern whereas the bulk d-PEO shows complete motional narrowing of the signal. This indicates that even though the local segmental motion of the intercalated d-PEO appears more dynamic at lower temperatures, the silicate layers still restrict motion such that some local configurations of the chain are not accessible and thus complete signal averaging is not possible.

Complementary spin-lattice relaxation measurements corroborate the observations made using the ${ }^{2} \mathrm{H}$ line-shape measurements. Based on these measurements the low temperature relaxation times are dramatically shorter in the intercalated sample as compared to the bulk, indicating enhanced polymer re-orientation dynamics in the intercalated samples. Furthermore, the temperature dependence of the relaxation time in the bulk and intercalated sample show dramatic differences. While the relaxation time for the intercalated sample passes smoothly from low to high temperatures, the bulk sample shows a break between the crystalline state and melt state, with the melt state relaxation times at least one order of magnitude faster than those observed in the intercalated sample at the same temperature.

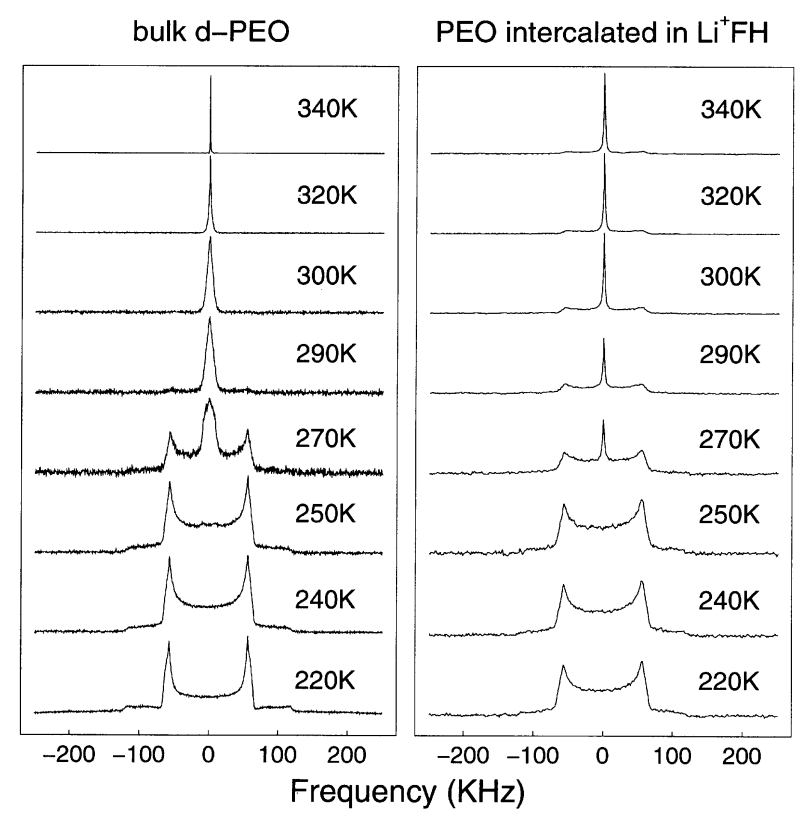

Fig. 13. Temperature dependence of ${ }^{2} \mathrm{H}$ NMR spectra, for bulk PEO and for PEO intercalated in $\mathrm{Li}^{+}$fluorohectorite. Adopted from [13]. 
The enhanced local chain dynamics of the intercalated d-PEO reflect the absence of chain entanglements and the presence of excess free volume associated with the packing constraints of intercalated chains. On average, the constraints to local chain dynamics on the length scale of a few monomers are less than those of the complementary chain in the bulk. However, constraints to local chain motion are not wholly absent, as indicated by the residual powder pattern at elevated temperatures. Based on geometrical considerations of the extremely narrow $0.8 \mathrm{~nm}$ interlayer, a large fraction of the intercalated PEO units are in direct contact with the surface of the silicate. Topologically, the surfaces of the opposing silicate layers will restrict the motions of the d-PEO chains. In addition, interactions of the relatively polar PEO segments with the polar silicate surfaces as well as with the interlayer cations will bias the local motion of the d-PEO chain to certain energetically favorable orientations. Because of the extended pseudo-2D nature of the chain and the absence of chain entanglements, the local dynamics of the intercalated chains may approach that of a single chain with no excluded volume effects.

Similar results of strong hindering of polymer mobility by surfaces have also been found by Brik et al. [37]. They studied the motional heterogeneity of PEO grafted between silica particles using electron paramagnetic resonance as well as NMR. They found that the organic phase exhibits marked variations in dynamic behavior with the liquid-like mobility of the polymer strongly hindered at the silica grafting nodes. Computer simulations probing the dynamics of confined oligomers and short polymers have indicated a strong influence of the confining surface on the local mobility of the molecules. Two modes are observed in these simulations - the presence of a fast mode corresponding to the 'tail' segments far away from the wall and a slow mode (whose slowing down depends on the surface interaction) corresponding to the 'train' segments close to the confining surface, we further discuss these simulation findings in the next section.

\subsection{3}

\section{Computer Simulations}

Computer simulations of nanoscopic confined fluids have revealed many details of the dynamics under confinement. The nature of the confined fluids - especially in the immediate vicinity of attractive surface - has been shown to be strongly altered by the confining surfaces, and this is manifested by a behavior dramatically different from the bulk fluids in the local relaxation [38a], the mobility [38c] and rheological properties [39] of molecules near adsorbing surfaces. For monomeric systems many computer simulation studies [40] provide a clear enough picture for the dynamics of confined films of small spherical molecules. On the other hand, for confined oligomers and polymers less has been done, especially towards the understanding of the dynamics of nanoscopic films [41].

Some of the pioneering work has focused on the behavior of abstract Lennard-Jones oligomers in nanoscopic confinements and consists of studies of the relaxation times of different 'modes' [38a,b,d], of the transport properties like the self-diffusion coefficients and the mean square displacements [38c,d], 
and of the rheological properties and the viscosity [39]. Although the oligomers used in these simulations are too short to exhibit genuine polymeric behavior they are characterized by very high flexibility. This renders the Rouse modes of short oligomers to be well defined near confining walls, when they are far apart ( $\sim 10$ monomer diameters $)$ to create two well separated and noninteracting fluid-wall interfaces [38a], as well as in thiner films (3-6 monomer diameters) [38d] - geometries which are comparable with the confining environment of the intercalated polymer. Moreover, the length of this oligomer is comparable to the average train size of high molecular weight polymers [42] and thus the dynamics of the adsorbed oligomers will mirror the dynamics of the adsorbed parts (trains) of much longer polymer coils in confinement [38a]. In these systems the end-to-end relaxation (first Rouse mode) is studied through the time correlation function of the end-to-end vector and its relaxation time is the rotational relaxation time of the chains. The time correlation function of the end-to-end vector is defined [41-44]:

$$
\begin{aligned}
<P(t) \cdot \boldsymbol{P}(0)\rangle & =\left\langle\left(\boldsymbol{R}_{N}(t)-\boldsymbol{R}_{0}(t)\right) \cdot\left(\boldsymbol{R}_{N}(0)-\boldsymbol{R}_{0}(0)\right)\right\rangle \\
& \sim \exp \left(-\frac{t}{\tau_{r}}\right), \quad t \geq \tau_{r}
\end{aligned}
$$

where $\boldsymbol{P}$ is the end-to-end vector, $N$ the number of beads in the chain, $\boldsymbol{R}_{i}(t)$ is the position of the $i$ bead of the chain at time $t$ and $\tau_{r}$ is the first Rouse mode relaxation time.

Using Eq. 1.2 the time correlation function of the end-to-end vector was calculated for the hexamer systems in slits of the same thickness as the interlayer gallery of PEO/fluorohectorite intercalated hybrids [38d: Section 2.2], for the whole film (Fig. 14a) and for the adsorbed chains (Fig. 14b). The film as a whole exhibits a multimodal relaxation, including fast relaxing and much slower relaxing species. The fast, bulk-like, relaxation is due to the free chains and the slower part is due to the superposition of the slower modes of the adsorbed chains and the relaxation times involved for each mode depend on the number of adsorbed segments (Fig. 15).

The most crucial parameter that determines quantitatively the relaxation of the confined chains is proved to be the wall affinity $\left(\varepsilon_{w}\right)$. Physically, $\varepsilon_{w}$ is the excess attractive energy per segment of the wall-polymer interaction, compared to the polymer-polymer interaction on a segmental basis. For neutral walls $\left(\varepsilon_{w}=0\right)$ the term 'adsorbed chain' is rather ambiguous, since the interaction between the solid particles and the fluid segments has no attractive part, and has only the meaning of chains with segments located inside the first fluid layer. In this sense, the chains touching the neutral walls $\left(\varepsilon_{w}=0\right)$ are in an environment analogous to the one felt by free chains in the $\varepsilon_{w}=2$ or $\varepsilon_{w}=3$ slits or by the free pentamers inside the second layer of a very wide pore. The effect of the neutral wall is a weak slowdown $\left(\tau_{\text {conf. }} \sim \tau_{\text {bulk }}\right)$ of the chain relaxations and the segment mobilities [38]. The main simulation findings [38] are as outlined below. 

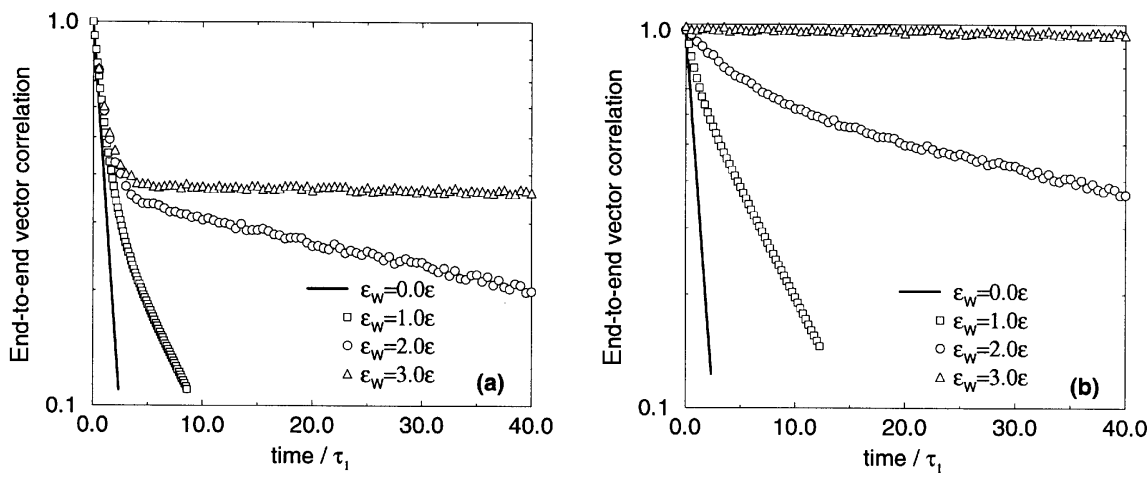

Fig. 14. End-to-end vector time correlation functions for various wall-polymer affinities (a) for the whole film (approximately same width as the intercalated PEO interlayer gap), (b) for the adsorbed chains independently of the number of contacts. Adopted from reference [38d].

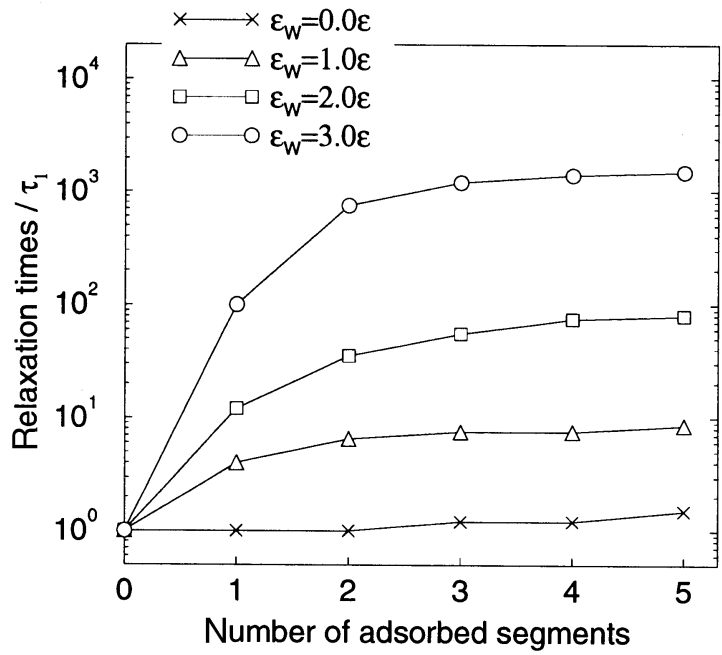

Fig. 15. Relaxation times of the first Rouse mode of confined pentamers $(\mathrm{h}=10)$ as a function of the number of contacts for all the simulated wall affinities, from Bitsanis I, Pan C (1993) J Chem Phys 99: 5520. The data indicate clearly a dramatic increase of the relaxation time inside the solid-oligomer interface with increasing $\varepsilon_{\mathrm{w}}$. The origin of these "glassy" dynamics is attributed to the slowdown of segmental motions inside the adsorbed layer. 
The relaxation times of the end-to-end vector time correlation function when calculated for the whole film (through the end-to-end vector time correlation function) exhibits a multimodal relaxation. The fast relaxation corresponds to the free chains located in the middle of the film and the slower modes to the relaxation of the adsorbed chains. This is concluded both by the relaxation times as calculated for those classes of chains separately, as well as by the ratio of the correlation function amplitudes of the fast versus the slow modes, which is very close to the ratio of the number of free versus adsorbed chains.

For the free chains the relaxation time is the same and very close to the bulk value. This happens even for the chains located inside the second fluid layer of wide gaps [38a] and the chains in much narrower confinements, where the space available for the free chains is comparable in size to the statistical segment.

The relaxation times of the adsorbed chains are higher compared to the bulk and vary significantly with wall affinity. The extent of the increase ranges from three orders of magnitude for the strongly physisorbing surfaces $\left(\varepsilon_{w}=3\right)$ to less than ten times for weakly physisorbing surfaces $\left(\varepsilon_{w}=1\right)$ and to almost a negligible degree $(\sim 1.3)$ for the neutral walls (Fig. 15).

The relaxation time of the end-to-end vector correlation for the adsorbed chains depends on the number of contacts. Chains with one or two contacts have most of their segments free and thus due to their bulk like dynamics the end-toend vector can rapidly relax. On the other hand, for the chains with most of their segments adsorbed this process becomes very slow as the segment dynamics are very sluggish inside the solid oligomer interface (Fig. 15). For strong wall attractions $\left(\varepsilon_{w}=2\right.$ or 3$)$ the chains with more than three contacts relax with almost the same time constant. This insensitivity shows that the slowdown of the dynamics is caused by the densification inside the first layer rather than the magnitude of the surface-fluid interactions [38a,d].

For more realistic molecular structures, that involve bond angles and tetrahedral potentials such as simulations of confined alkane oligomers [43], similar behavior has been observed. There is a co-existence of fast and slow relaxing species, which were identified as the free and adsorbed molecules respectively, and for strongly physisorbing surfaces the first Rouse mode, as well as trans-to-gauche transition dynamics, slowed down by three orders of magnitude in comparison with the equivalent bulk. Moreover, the confined alkanes create liquid crystalline domains with smectic ordering and exhibit quite high diffusivities parallel to the confining surface, despite the huge slow down of their relaxation times [43a].

\subsection{4}

\section{Cooperative Motion - TSC and DSC Measurements}

The presence of cooperative motion of chain segments present in intercalated polymer chains can be examined using various analytical techniques such as Differential Scanning Calorimetry (DSC), thermally stimulated current (TSC) and dielectric spectroscopy. DSC measurements on an intercalated PEO, $\left(M_{w}=\right.$ $100,000) /$ montmorillonite hybrid ( $20 \mathrm{wt}$ \% polymer), indicated the absence of 
any thermal transitions corresponding to the glass or the melting transition of PEO $\left(\mathrm{T}_{\mathrm{g}} \sim-55^{\circ} \mathrm{C}\right.$ and $\left.\mathrm{T}_{\mathrm{m}} \sim 65^{\circ} \mathrm{C}\right)$. DSC studies of polystyrene intercalated in an organically modified layered silicate (Fig. 16) also indicated that the intercalated hybrid does not show a thermal transition corresponding to the glass transition of polystyrene over a temperature range of $50-180^{\circ} \mathrm{C}$ [45]. Similar absence of a glass transition for polystyrene confined to zeolite cavities have also been observed by Frisch and coworkers [46].

Keddie and coworkers [47] have investigated the glass transition temperatures as a function of film thickness for thin films of polystyrene and poly(methyl methacrylate) on different substrates. For polystyrene on $\mathrm{Si}(111)$, they observed a decrease in the glass transition temperature $\left(\mathrm{T}_{g}\right)$ for films thinner than $400 \AA$ independent of molecular weight. This decrease was attributed to the presence of a liquid-like layer at the free surface of the film. Similar results were also reported by Keddie et al. for PMMA on gold surfaces, wherein the surface polymer interaction is expected to be again weak. However, in the presence of a strongly interacting surface, the glass transition was found to dramatically increase with decreasing film thickness and was attributed to the presence of a layer near the surface wherein the mobility is greatly reduced [48] in agreement with computer simulation studies [49].

DSC measurements possess a relatively low sensitivity to weak glass transitions. This is particularly exaggerated in the case of the intercalated compounds

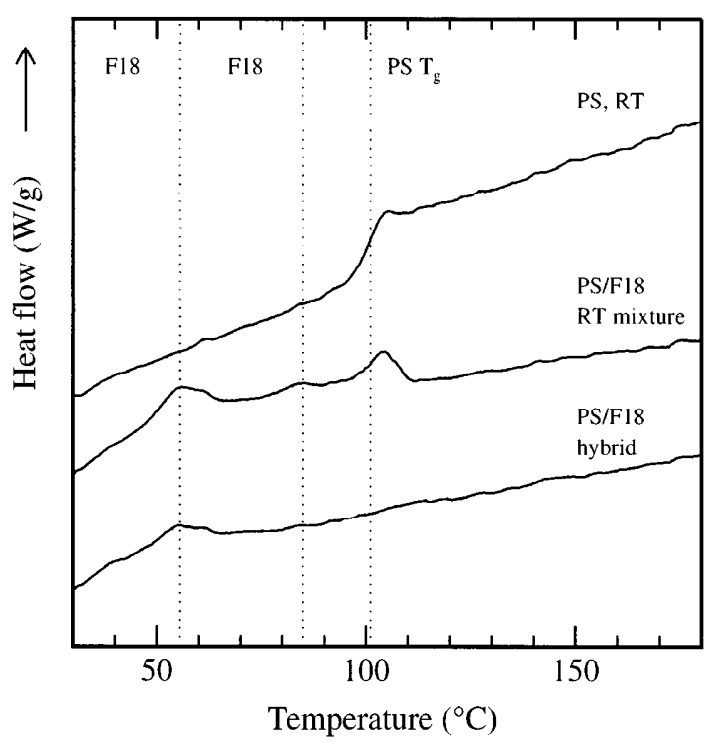

Fig. 16. DSC measurements of a polystyrene/C18FH intercalated hybrid. For comparison DSC traces for the neat polymer and for a physical mixture of the polymer and the organically modified silicate are also shown. There is an absence of any thermal transition for the intercalated polymer around the bulk $\mathrm{T}_{\mathrm{g}}[45]$. 
where there is a low volume fraction of the polymer. TSC techniques, on the other hand, have enhanced sensitivity to cooperative relaxations such as glass transitions [50]. To further evaluate the glass transition region of the intercalated PEO TSC was applied in two modes - 'global' and 'thermal sampling'. Due to the small dipole associated with polystyrene, similar measurements could not be performed with the intercalated polystyrene system. The 'global' TSC spectra have some similarity to a.c. dielectric loss spectra. As with the DSC measurements, no clear peak was observable in the global TSC spectra of the intercalated PEO in the temperature range of the bulk glass transition for the intercalated PEO. Thus, cooperative relaxations of the intercalated polymer are, at best, weak.

TSC measurements with 'thermal sampling' are very sensitive to cooperative relaxations even from a minor fraction of the overall relaxing species. Glass transition regions can be resolved using this method even if the relaxations are extremely broad or weak. The apparent activation energies, $\mathrm{E}_{\mathrm{a}}$, of relaxations contributing to the depolarization current for $100 \%$ PEO, the $20 \%$ PEO melt intercalate, and the $0 \%$ PEO pressed montmorillonite control pellet are shown in Fig. 17. The analysis consists of assigning cooperative glass transition like motions to the regions of departure of the values of $\mathrm{E}_{\mathrm{a}}$ from the $\Delta S^{\ddagger}=0$ prediction, where $\Delta S^{\ddagger}$ is the activated state entropy. For non-cooperative 'secondary' transitions it has been observed that $\Delta S^{\ddagger}=0$. A single sharp peak, typical of amorphous and semicrystalline polymers, is seen at the $\mathrm{T}_{g}$ for the $100 \%$ PEO sample. However, for the intercalated PEO nanocomposite a distinct peak in $\mathrm{E}_{\mathrm{a}}$ is not observed. Instead, a broad transition starting at about the nominal $\mathrm{PEO} \mathrm{T} \mathrm{T}_{g}$ and ranging up to about $60^{\circ} \mathrm{C}$ is seen.

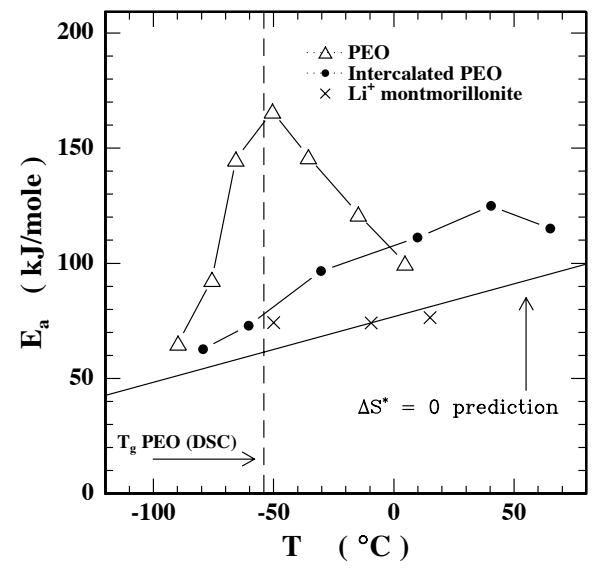

Fig. 17. Temperature dependence of the apparent activation energies $E_{a}$ as determined by TSC-TS. Bulk PEO, the neat montmorillonite silicate and an intercalated $20 \mathrm{wt}$. \% $\mathrm{PEO} /$ montmorillonite hybrid are shown. The departure of $\mathrm{E}_{\mathrm{a}}$ from the $\Delta \mathrm{S}^{\ddagger}=0$ prediction line indicates the glass transition region. Figure adopted from [14]. 
Since no melting transition was observed in DSC, the fraction of 'amorphous' PEO chains in the intercalate is similar to the total PEO content of the hybrids (in this case $\sim 20 \%$ ). Therefore, the relaxation strength should be at a level where we can easily detect the 'cooperative' motions. However, the cooperative relaxations detected in the glass transition region (Fig. 17) are weaker than any measured before using the TSC-TS technique. Of the several systems studied with the TSCTS technique, never before has such a broad transition region with a low degree of cooperatively been observed [50,51]. Based on the data in Fig. 17, it was concluded that the motions of the intercalated PEO chains are inherently non-cooperative relative to the cooperative $\mathrm{T}_{g}$ motions in less unconstrained environments such as the amorphous portion of the bulk polymer or the amorphous chains confined between crystal lamellae in pure semi-crystalline PEO. For semi crystalline PEO, the chains are confined in 'amorphous' gaps a few nanometers wide whereas the intercalated PEO occupies a gap of less than $1 \mathrm{~nm}$ !

Taken in context with previous investigations of polymer relaxations in confined environments the results from these polymer intercalates appear to indicate that cooperative motion precipitously decreases as polymers are confined to extremely narrow slits less than a few nanometers.

\section{4}

\section{The Rheology of Polymer/Silicate Nanocomposites}

Rheology of various polymer layered-silicate nanocomposites - intercalated, exfoliated and end-tethered exfoliated (prepared by in-situ polymerization from reactive groups tethered to the silicate surface), have been performed in a conventional melt-state rheometer in both oscillatory and steady shear modes. These experimental studies have provided insight into the relaxation of polymer chains when confined by the layers of inorganic silicates, as well as the role of shear in orienting the layered nanocomposites.

\section{1}

\section{Linear Viscoelasticity}

The steady-shear rheological behavior of a series of intercalated poly(dimethyl $_{0.95}$-diphenyl ${ }_{0.05}$ siloxane)-layered silicate (dimethyl ditallow montmorillonite) nanocomposites (with varying silicate loadings) are shown in Fig. 18 [52]. The viscosity of the nanocomposites is enhanced considerably at low shear rates, and increases monotonically with increasing silicate loading (at a fixed shear rate). Furthermore, the intercalated nanocomposites display a shear-thinning behavior at low shear-rates, where the pure polymer displays a shear rate independent viscosity. At high-shear rates where the polymer displays shear-thinning behavior, the nanocomposites also display shear-thinning with the values of the viscosity (at least at the lower loadings of silicate) being comparable to that of the polymer itself. The same trends are also observed in linear dynamic oscillatory shear measurements, where the storage and loss moduli $\left(G^{\prime}\right.$ and $G^{\prime \prime}$ 


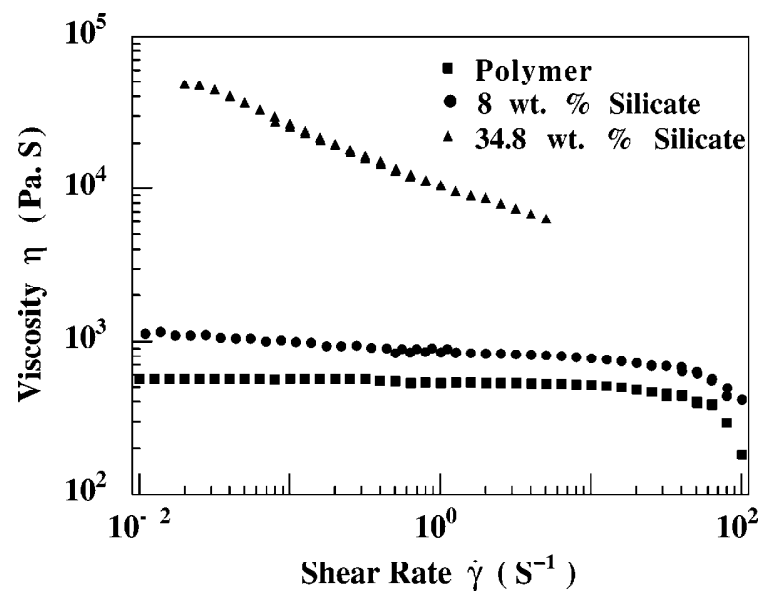

Fig. 18. The steady-shear rheological behavior for a series of intercalated nanocomposites of poly(dimethyl ${ }_{0.95}$-diphenyl ${ }_{0.05}$ siloxane) with layered silicate (dimethyl ditallow montmorillonite) at $25^{\circ} \mathrm{C}$. The silicate loading is varied and are noted in the legend. From Ref. [5].

respectively) exhibit a plateau at low-frequencies. Furthermore, consistent with the steady shear measurements, a monotonic increase in both $G^{\prime}$ and $G^{\prime \prime}$ at all frequencies is observed with increasing silicate content. It thus appears that the intimate contact between the polymer and the inorganic sheets leads to an alteration of the relaxation dynamics of the polymer, leading to the low-frequency plateau in the moduli and the low-shear rate non-Newtonian viscosity behavior.

In contrast, for a series of polydimethylsiloxane based delaminated hybrids (dimethyl ditallow montmorillonite), the steady-shear viscosity shows an increase with respect to that of the pure-polymer at low shear rates but still obeys Newtonian type behavior, even at the highest silicate loadings examined (Fig. 19). The increase in viscosity at the silicate loadings examined was roughly linear at all shear rates. Similar effects are also observed in linear dynamic oscillatory shear measurements, where the storage and loss moduli for the delaminated hybrids display similar frequency dependence as the pure polymer, with a monotonic increase in the magnitude of the moduli with increasing silicate loading [52]. It thus appears that in these delaminated hybrids, the relaxation of the polymer chains is not altered (at least within the sensitivity of the measurements) by the presence of the silicate layers. This rheological response is similar to the relaxation behavior of typical filled-polymer systems, wherein the relaxation dynamics of the polymer chains are not significantly altered by the presence of non-interacting filler particles [53].

In addition, two end-tethered delaminated hybrid systems prepared by insitu polymerization - (a) Poly ( $\varepsilon$-caprolactone)-montmorillonite (PCLC) and (b) nylon-6-montmorillonite $(\mathrm{NCH})$ - wherein the polymer chains are end-tethered to the silicate surface via cationic surfactants [54] (Fig. 20), were also studied. 


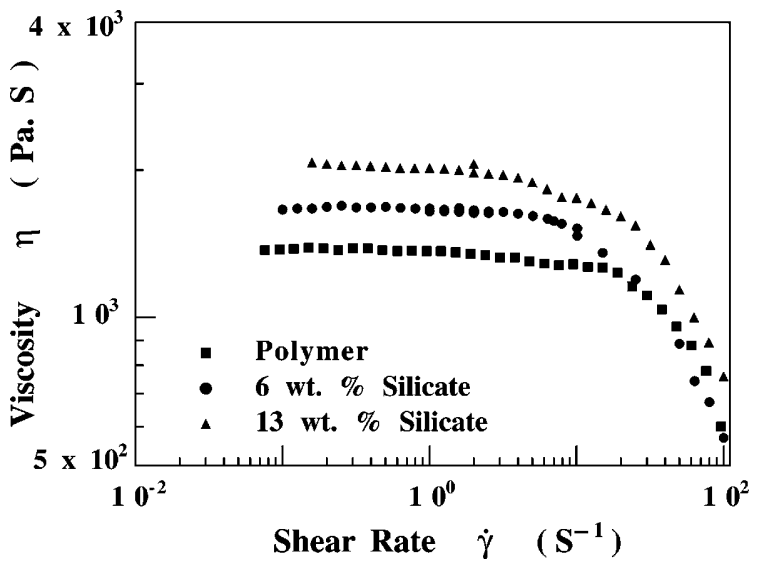

Fig. 19. The steady-shear rheological behavior for a series of delaminated nanocomposites of polydimethylsiloxane with dimethyl ditallow montmorillonite at $25^{\circ} \mathrm{C}$. The steady-shear viscosity shows an increase with respect to that of the pure-polymer at low shear rates but still obeys Newtonian type behavior, even at the highest silicate loadings examined. From Ref. [5].

The linear viscoselastic properties of the pristine nanocomposites, the effect of large amplitude oscillatory shear on the orientation of the nanocomposites and the viscoelastic properties of these oriented nanocomposites were examined.

The changing molecular weight of the polymer chains, a result of the synthetic scheme used in the preparation of the nanocomposites, poses some problems in the interpretation of the variation of the moduli with increasing silicate content. The average molecular weight decreased sharply at low silicate loadings and remained roughly constant for nanocomposites with more than $2 \mathrm{wt} . \%$ silicate. The linear viscoelastic response as measured by dynamic oscillatory measurements and quantified by $G^{\prime}$ and $G^{\prime \prime}$ for the five poly ( $\varepsilon$-caprolactone) based nanocomposites examined is shown in Figs 21 and 22 respectively. Data were acquired at several temperatures using the lowest possible strain amplitudes (typically in the range of $0.1-5.0 \%$ ) and shifted using the time-temperature superposition principle to form the master-curves presented in Figs 21 and 22. For all the nanocomposites examined, the data was somewhat restricted due to the alignment of the silicate layers by the application of large amplitude oscillatory shear (particularly required at low frequencies, to obtain force signals larger than the low limit of the transducer). This was significantly restrictive for the high silicate loading composites (PCLC5 and PCLC10) where at high temperatures and low frequencies, alignment of the layers would start to occur (as measured by a change in the rheological response consistent with alignment of the layers) at strain amplitudes as low as $10 \%$. Only data verified to be in the linear regime are shown in Figs 21 and 22.

The storage moduli (Fig. 21) for the nanocomposites show a monotonic increase at all frequencies with increasing silicate content, with the exception of PCLC2, where at the highest frequencies, it has a slightly lower value than PCLC1. The loss moduli (Fig. 22), on the other hand, show a somewhat non-mo- 


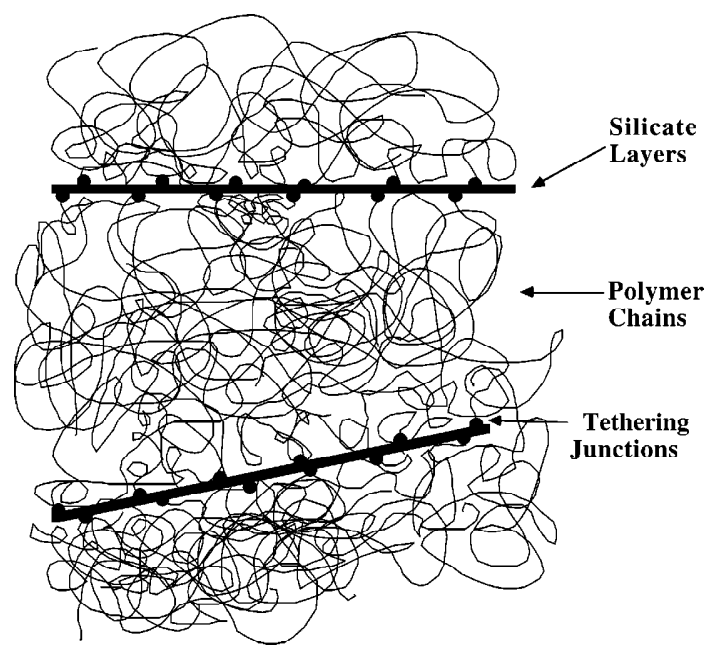

Fig. 20. Schematic diagram describing the end-tethered nanocomposites. The layered silicates are highly anisotropic with a thickness of $1 \mathrm{~nm}$ and lateral dimensions (length and width) ranging from $\sim 100 \mathrm{~nm}$ to a few microns. The polymer chains are tethered to the surface via ionic interactions between the silicate layer and the polymer-end. Adapted from Ref. [54].

notonic dependence, with the value for PCLC1 exceeding that for PCLC2, PCLC3 and PCLC5. However, the trend for the $2-10$ weight \% samples suggests that G" increases with increasing silicate loading. It is worthwhile to note the trend ofdecreasing molecular weight of the poly( $\varepsilon$-caprolactone)with increased silicate loading, with the largest decrease occurring from 1 to 2 weight $\%$ silicate loading, while the molecular weight of the polymer chains in the $2,3,5$ and $10 \%$ nanocomposites is nearly the same.

For the molecular weights of the PCL samples examined here, it is expected that at the temperatures and frequencies at which the rheological measurements were carried out, the polymer chains should be fully relaxed and exhibit characteristic homopolymer like terminal behavior i.e., $\mathrm{G}^{\prime} \sim \omega^{2}$ and $\mathrm{G}^{\prime \prime} \sim \omega$ (based on the relaxation behavior of pure PCL, the data presented in Figs 21 and 22 are those corresponding to frequencies below the cross-over frequency associated with the transition from the plateau to the terminal relaxation of the polymer). While the polydispersity of the polymer chain lengths would affect this behavior, the effect is expected to be small in the dynamic regimes probed. The highfrequency-regime frequency dependence of the storage modulus decreases monotonically with increased silicate loading from $\omega^{1.65}$ for PCLC1 to $\omega^{0.5}$ for PCLC5.

The frequency dependence of $\mathrm{G}^{\prime \prime}$ also progresses monotonically with silicate loading from $\omega^{1.0}$ for PCLC1 to $\omega^{0.65}$ for PCLC5. The frequency shift factors for the PCLC samples appear to be independent of the silicate loading consistent with the results for the frequency shift factors previously obtained for PCL [54]. 


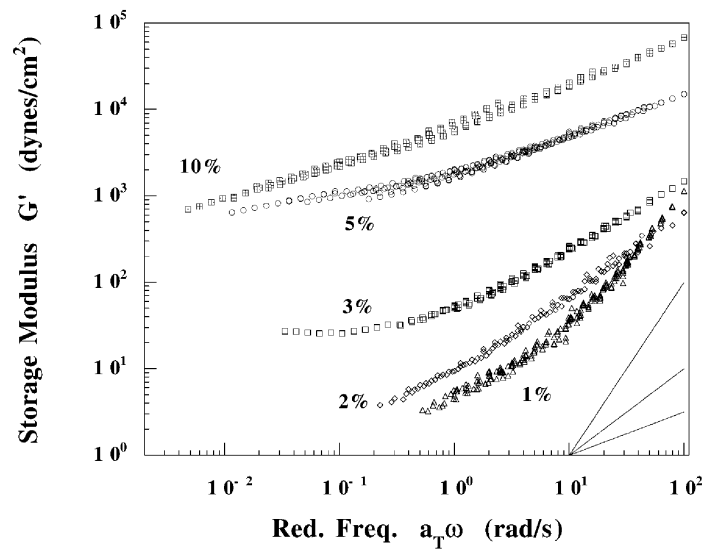

Fig. 21. Storage modulus ( $\left.G^{\prime}\right)$ for PCL based silicate nanocomposites. Silicate loadings are indicated by percentual values in the figure. Master-curves were obtained by application of time-temperature superposition and shifted to $\mathrm{T}_{0}=55^{\circ} \mathrm{C}$. From Ref. [54].

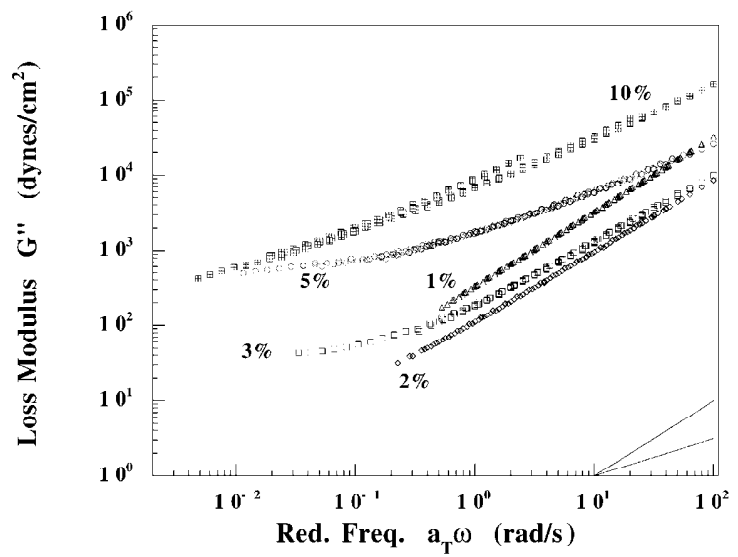

Fig. 22. Loss modulus $\left(G^{\prime \prime}\right)$ for PCL bases silicate nanocomposites. The system is as described in Fig. 21. From Ref. [54].

Due to the low $\mathrm{T}_{g}$ of PCL $\left(\sim-60^{\circ} \mathrm{C}\right)$ and the measurements being performed at temperatures greater than $55^{\circ} \mathrm{C}$ (due to the crystallization of PCL), the temperature-dependent frequency shift factors are relatively small. The flow activation energy obtained by fitting the data at all silicate loadings is estimated to be 19 $\mathrm{kJ} / \mathrm{mol}$. Measurements on a higher molecular weight PCL homopolymer over the same temperature range yielded a value of $17 \mathrm{~kJ} / \mathrm{mol}$, in good agreement 


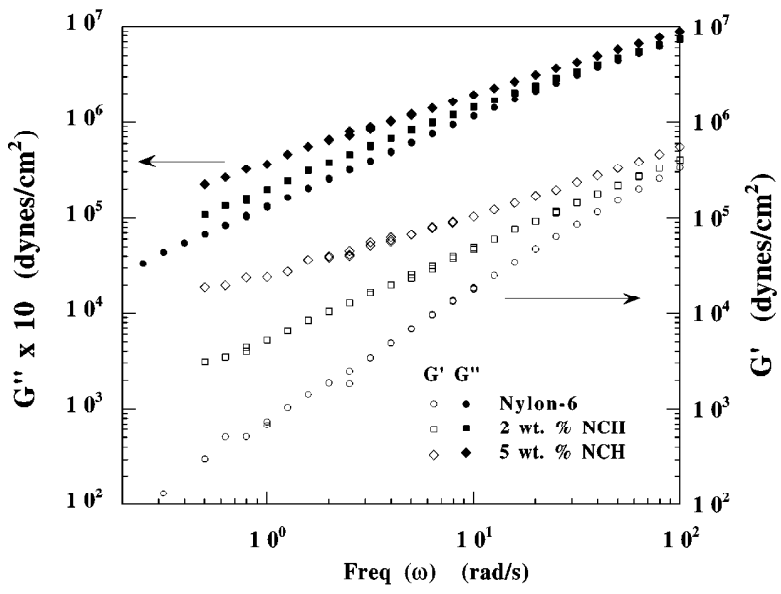

Fig. 23. Storage Modulus ( $\left.G^{\prime}\right)$ and Loss modulus $\left(G^{\prime \prime}\right)$ for nylon-6 silicate hybrids. Silicate loadings are indicated in the figure. All measurements were carried out at a single temperature of $235^{\circ} \mathrm{C}$. From Ref. [54].

with the results for the PCL nanocomposites. This implies that the temperature dependence of the relaxation being probed is that of the polymer. Since the silicate layers to which the polymers are tethered do not have a temperature-dependent relaxation, the only relaxation process probed as a function of temperature is that of the polymer segments. While the tethering must have some influence on the local dynamics of the monomers near the tethering junction, the temperature dependence of the rheological properties appears to indicate that the influence of the surface and the junction appear to be extremely local.

Similar rheological behavior was also observed with the nylon-6 silicate hybrids produced by Ube Chemical Company (Fig. 23). G' and G" recorded at $235^{\circ} \mathrm{C}$ for a pure nylon-6, a 2 weight $\%$ nylon- 6 nanocomposite and a 5 weight $\%$ nylon-6 nanocomposite [5] are shown. Both $\mathrm{G}^{\prime}$ and $\mathrm{G}^{\prime \prime}$ exhibit a monotonic increase with increasing silicate loading at all frequencies. Unlike the PCL nanocomposites, the molecular weight of the polymer matrix is nearly the same for all three nylon samples. This result further suggests that the inconsistency observed in the PCL nanocomposites with the 1 weight \% sample showing a higher $\mathrm{G}^{\prime}$ value than that for the 2 weight $\%$ sample may be caused by the abrupt decrease in molecular weight in the latter sample.

In close analogy to the PCL based nanocomposites, the terminal zone dependence of $G^{\prime}$ and $G^{\prime \prime}$ for the 2 and 5 weight $\%$ samples, show non-terminal behavior with power-law dependencies for $\mathrm{G}^{\prime}$ and $\mathrm{G}^{\prime \prime}$ much smaller than the expected 2 and 1 respectively. Furthermore, like the PCL based nanocomposites, there also appears to be a gradual decrease in the power-law dependence of $\mathrm{G}^{\prime}$ and $\mathrm{G}^{\prime \prime}$ with increasing silicate loading.

The linear viscoelastic measurements of the end-tethered nanocomposites reveal several features unique to these materials. Time-temperature superposi- 
tion can be applied to the low-amplitude oscillatory shear rheological response, with temperature shift factors being similar to that of the pure polymer. Holding the molecular weight relatively constant (the nylon-6 series and PCLC2 PCLC10) reveals that at any given frequency the magnitude of the storage and loss moduli increase monotonically with increasing silicate loading. Furthermore, the frequency dependence of the high-frequency behavior of $G^{\prime}$ and $G^{\prime \prime}$ shows a gradual change with increasing silicate loading - from homopolymerlike behavior at low silicate loading to increasingly non-terminal behavior at higher silicate contents and finally plateauing at $G^{\prime}$ and $G^{\prime \prime} \sim \omega^{0.5}$ for silicate loadings of greater than 5 weight \%. Finally, at the very lowest frequencies accessed, these nanocomposites exhibit a low-frequency behavior that has both $\mathrm{G}^{\prime}$ and $\mathrm{G}^{\prime \prime}$ almost independent of frequency.

The frequency dependence of $\mathrm{G}^{\prime}$ and $\mathrm{G}^{\prime \prime}$ for these end-tethered nanocomposites can be contrasted to those observed in a series of delaminated hybrids with no end-tethering. Non-terminal flow behavior has been observed in filled-polymer systems exhibiting yield phenomena, but only in cases wherein the filler and polymer are actively interacting and in a dynamic regime controlled by much larger length scales (i.e., lower frequencies) than those observed in this study. Furthermore, the deviations from homopolymer-like behavior in these systems have been observed at relatively high filler-loadings. At similar high silicate loadings in either exfoliated or intercalated hybrids (not tethered to the silicate surface), significant non-linear rheological behavior is observed thereby complicating the data analysis [55]. The analogies with filled polymer systems may have particular relevance to the low-frequency behavior of the storage modulus in the PCLC nanocomposites and are discussed in greater detail below.

Non-terminal low-frequency rheological behavior has also been observed in ordered block copolymers and smectic liquid-crystalline small molecules $[56,57]$. Several hypotheses have been suggested to explain the observed rheological behavior in these systems [56-62]. Koppi et al. have suggested that undulations and defects in the layers might contribute to the low-frequency viscoelastic response in layered block copolymers [56]. Other ideas include that the domain structure of the ordered mesophases is responsible, due to the dynamic processes on both the microscopic and mesoscopic length scales. It is also well documented that topological defects also affect rheological properties dramatically, particularly in the low-frequency terminal regime [57]. Effects of domain collective dynamics were studied by Kawasaki and Onuki [58], who demonstrated that overdamped second-sound modes in an orientationally disordered lamellar phase could result in anomalous low-frequency rheological behavior. Rubinstein and Obukhov [59] also obtained the same result by considering diffusion-controlled annihilation of defects in a disordered lamellar system. The results of Larson et al. [57] with smectic small molecules and short ordered block copolymers has suggested that the non-terminal low-frequency response is due to the long-range domain structure and the presence of defects.

The silicate layers in the hybrids are highly anisotropic with lateral dimensions ranging from 100 to $1000 \mathrm{~nm}$, and even when separated by large distances 
(i.e., when delaminated) cannot be placed completely randomly in the sea of polymer (as seen in Fig. 20). Furthermore, the majority of the polymer chains in the hybrids are tethered to the surface of the silicate layers. Thus, it can be expected that there are domains in these materials, even above the melting temperature of the constituent polymers, wherein some long-range order is preserved and the silicate layers are orientated in some preferred direction. Furthermore, this long-range order and domain structure is likely to become better defined at the higher silicate contents, where the geometrically imposed mean distance between the layers becomes less than the lateral dimensions of the silicate layers and thus forcing some preferential orientation between the layers. However, there is likely to be considerable polydispersity effects in terms of the orientation and the distance between the silicate layers. Many such randomly oriented grains make up the entire sample leading to the presence of a macroscopically disordered material. Thus in general, the material possesses a layered structure, with grains wherein the silicate layers are oriented in a preferred direction leading to the presence of grain boundaries and the concomitant presence of defects. However, for delaminated hybrids with no end-tethering, an increase in the moduli at all frequencies is observed and classical homopolymer like terminal behavior. Hence it appears that delamination alone is not sufficient to produce the non-terminal flow behavior.

The non-terminal low-frequency behavior observed in the PCL and nylon-6 based nanocomposites could also be attributed to the retardation of molecular relaxation processes produced by the tethering of one-end of these molecules to the silicate surface. Witten et al. [62] have suggested that tethering of the polymer molecules is expected to create an energetic barrier to the reptation motion, which leads to a dramatic increase in the relaxation time and hence a shift of the terminal relaxation to very low frequencies. Since the molecular weight of the samples examined here is small, it is expected that the dominant relaxation mode would be Rouse-like, which should not be drastically slowed by the tethering of one-end of the chains. Furthermore, at all silicate loadings, most of the chains are tethered to the silicate layers and any effect of the tethering should be discernible at all loadings [63]. However, it is clear that the terminal-zone behavior gradually changes with increased silicate loading, saturating at about 5 weight $\%$.

The presence of a transition is manifested from the change of slope observed at high frequency to a more flattened behavior at low frequencies. The change is more pronounced in the case of $G^{\prime}$ than $G^{\prime \prime}$. The low frequency response is indicative of a 'pseudo solid-like' behavior and is clearly seen in the PCL samples with silicate loading greater than 3 weight $\%$. Similar rheological response at low-frequencies has been observed in triblock copolymers in the ordered state and has been attributed to the quasi-tethering of the unlike polymer segments in their respective microdomains [64]. A solid-like response has also been observed in conventionally filled polymer systems in which there were strong interactions between the polymer and the filler and has been attributed to the presence of yield phenomena in these systems [65]. Thus, the presence of the silicate layers and the lack of complete relaxation of the chains contribute to the 
pseudo solid-like response at low frequencies (pseudo solid-like as $\mathrm{G}^{\prime}$ does not exceed $G^{\prime \prime}$ by orders of magnitude as would be expected from a true solid).

\section{2}

\section{Alignment of Nanocomposites}

Application of large strain-amplitude oscillatory shear leads to a shear-aligned sample. These measurements were carried out on the 3,5 and $10 \%$ samples and the during-shear moduli show a decrease with continual shearing and finally reach a plateau value. With the exception of the first few cycles of shear for the 3 and 5 weight $\%$ samples, the modulus decreases monotonically and the stress signal remains sinusoidal. In the first few cycles of shear for the 3 and 5 weight $\%$ samples, the moduli show a maximum, before monotonically decreasing. The small-strain moduli after shear-alignment for PCLC10 carried out at T=70 ${ }^{\circ} \mathrm{C}, \omega$ $=1 \mathrm{rad} / \mathrm{s}, \gamma_{\mathrm{o}}=120 \%$, and time $=3 \mathrm{~h}$ are shown in Fig. 24 . First, both the storage and loss moduli for the aligned sample are considerably lower than those for the initially unaligned sample. Secondly, the frequency dependence of both $G^{\prime}$ and $G^{\prime \prime}$ for the aligned samples are much stronger and start to resemble those of free homopolymers. The small-strain modulus results observed for the PCLC samples before and after shear alignment are in close analogy with those observed for block copolymers as well as small molecule smectic liquid-crystals [56,57]. The temperature-dependent frequency-shift factors for the aligned and initially unaligned sample are within experimental errors identical. That large-amplitude oscillatory shear can significantly alter the (small-strain) linear viscoelastic

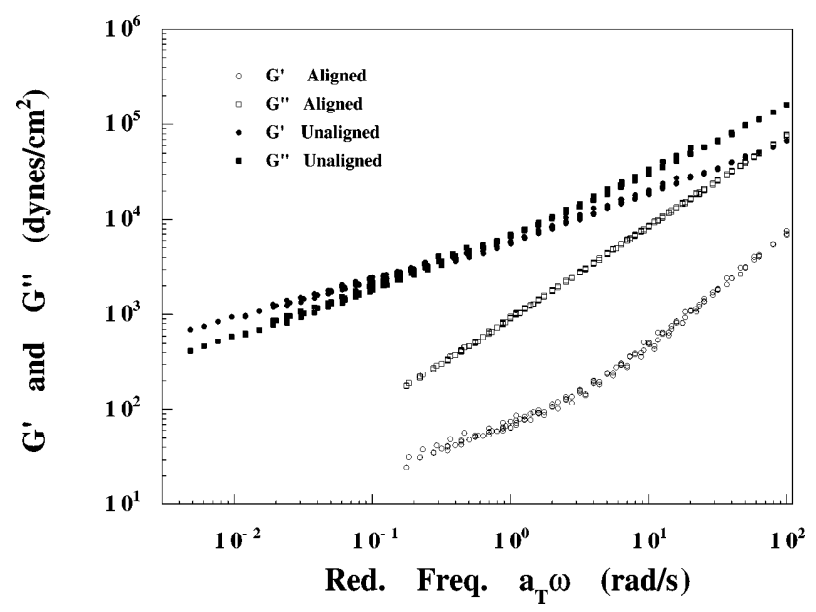

Fig. 24. G' and G" obtained using small amplitude oscillatory strain for PCLC10 before and after large-amplitude shear. See text for conditions of large amplitude shear. Master curves obtained by application of time-temperature superposition and shifted to $\mathrm{T}_{0}=55^{\circ} \mathrm{C}$. From Ref. [54]. 

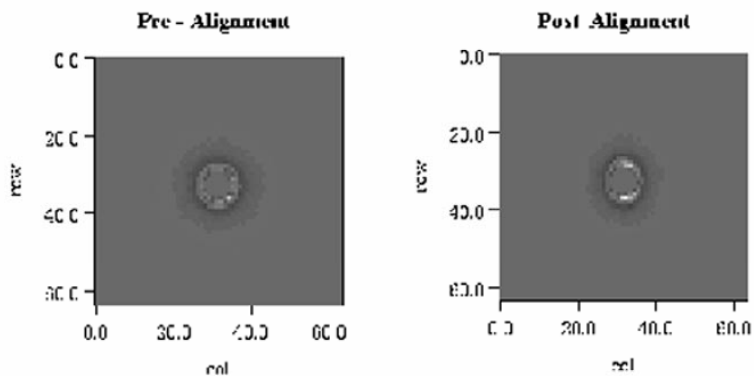

Fig.25. Preliminary SANS measurements on post large amplitude oscillatory shear confirm the presence of global alignment of the silicate layers.

response, indicates that there is some mesoscopic arrangement of the silicate layers, which is organized by the application of large amplitude shear. Preliminary SANS measurements on post large amplitude oscillatory shear confirm the presence of global alignment of the silicate layers as seen in Fig. 25. Similar alignment effect has been also observed in injection molded nylon- 6 hybrids as observed by the Toyota group. TEM and SAXS measurements on extruded nanocomposites revealed highly oriented samples at the edges, with relatively poorer order in the region of lowest shear, i.e. the central plane.

\section{3}

\section{Strain - Hardening of Polymer Brushes}

Strain sweeps at fixed frequencies $(\omega)$ and temperature were carried out on all aligned samples. Complex viscosity $\left(\eta^{\star}\right)$ and phase angle $(\tan \delta)$ as a function of strain amplitude for PCLC10 at $\omega=1 \mathrm{rad} / \mathrm{s}$ and $3 \mathrm{rad} / \mathrm{s}\left(\mathrm{T}=55^{\circ} \mathrm{C}\right)$ during both increasing and decreasing strain amplitude cycles are shown in Fig. 26. At all strain amplitudes the torque was confirmed to be sinusoidal, thereby allowing for interpretation in terms of standard viscoelastic parameters. The complex viscosity at low strain amplitudes is independent of strain amplitude and is found to be dominated by the viscous response. However, progression to higher strain amplitudes leads to an increase in $\eta^{\star}$ with the elastic component becoming more prominent as seen by the decrease in the $\tan \delta$. At the highest strain amplitudes $\eta^{*}$ appears to saturate, with a value much higher than that observed at low strain amplitudes. A slight hysteresis in the transition from high to low viscosity is also observed when the strain amplitude is decreased from a large value to a small value (as compared to the case where the strain amplitude is increased from low to high values).

Similar results were obtained on all three samples over a range of frequencies and temperatures. The upturn in the viscosity (and the downturn in $\tan \delta$ ) can be seen to occur at a critical strain amplitude over a wide range of temperatures and frequencies (for a given silicate loading). Three important features are observed in the rheological response of all three samples - (a) the process is reversible; (b) there 

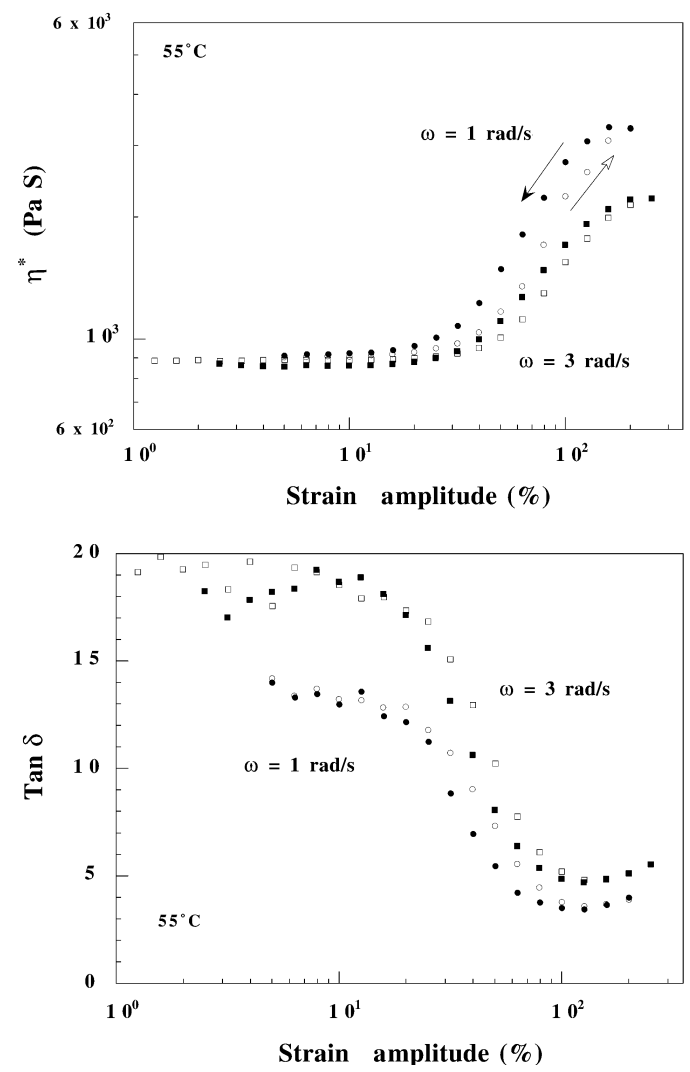

Fig. 26. Complex viscosity $\left(\eta^{\star}\right)$ and phase angle $(\tan \delta)$ as a function of strain amplitude for PCLC10 at $\omega=1 \mathrm{rad} / \mathrm{s}$ and $\omega=3 \mathrm{rad} / \mathrm{s}\left(\mathrm{T}=55^{\circ} \mathrm{C}\right)$ during both increasing and decreasing strain amplitude cycles. Open symbols were obtained with increasing strain amplitude and filled symbols with decreasing strain amplitude.

is a critical strain amplitude for the transition; and (c) the elastic component to the rheological response becomes more important with increasing strain amplitude.

Typically homopolymers in a shear flow exhibit decreasing viscosity with increasing shear rate. Also, tethered polymer solutions in a surface force apparatus, shear thin at a critical velocity presumably due to the slip of the tethering mica-layers [66]. These systems have also shown a dramatic increase in normal force beyond a critical Weissenberg number, and this has been attributed to hydrodynamic instabilities, flow induced brush thickening as well as shear induced diffusion [67,68]. In contrast, in these aligned end-tethered nanocomposites we observe an increase in viscosity with increasing shear strain amplitude with the transition occurring at modest strain amplitudes.

Based on the density of grafting, the systems examined are expected to be in the 'strong' brush regime, wherein the chains are strongly stretched away from the tethering surface, even in the absence of any external force. Upon application 
of shear-strain (beyond the critical strain amplitude), the tethered polymers are expected to completely unwind in response to the applied shear [69-72]. Based on the limitations of a 'melt-brush' system, wherein the tethered polymer has to fill all space, the chains stretch. This stretching occurs at a critical displacement that only depends on the geometrical restrictions of the system of interest i.e., the distance between tethering surfaces. This is borne out in the observation that the critical displacement decreases with increasing silicate content. It is expected that as the spacing between layers is decreased, the equilibrium chain structure is further distorted, and therefore the critical shear displacement required for the chain stretching is decreased. This is an unexpected result in light of solution based experiments, where the systems exhibit a decrease in viscosity beyond a critical velocity and have been theoretically attributed to the disentangling of chains from adjacent tethering surfaces and consequent slip of the layers. Further, mechanisms such as shear induced diffusion or brush thickening would require a frequency/temperature dependence to the observed phenomena and are also predicated on the presence of space filling solvent. The experimental results of the melt polymer brush system clearly show that the mechanism of flow in a melt brush are dramatically different than in solution-brushes and these effects manifest as opposite rheological responses with the controlling parameter for the transition being a critical displacement for the melt-brushes as opposed to a critical velocity for the solution-brushes [73].

Doyle et al [67] have performed Brown ian dynamics simulations of solvent filled polymer brushes under steady and oscillatory shear flows. Under steady flow they observe shear thinning behavior for both the normal force and the shear viscosity. Under oscillatory flow, they found extreme shear thickening when the ratio of the Weisenberg number to the frequency $\sim 3$. They believe that shear induced diffusion is the cause for this shear thickening. They also find the behavior to be more predominant at high frequencies, where the molecules of the chain effectively do not feel the tethering sites and can hence move affinely. Due to this affine motion the molecules collide with the neighbors and experience an effect similar to shear-induced diffusion as seen in theory of free suspensions. While this interpretation is useful in explaining some of the anomalous rheological responses of polymer brushes in a solvent, studied by the surface forces apparatus, it is clearly incapable of physically describing the dynamical processes observed in melt brushes [73].

\section{5}

\section{Conclusions}

Polymer silicate nanocomposites offer unique possibilities as model systems to study confined polymers or polymer brushes. The main advantages of these systems are: (a) the structure and dynamics of nanoconfined polymer chains can be conveniently probed by conventional analytical techniques (such as scattering, DSC, NMR, dielectric spectroscopy, melt rheology); (b) a wide range of different polymers can be inserted in the interlayer or end-grafted to the silicate 
surfaces, through proper surface modification; and (c) since the confining surfaces are atomically smooth and the interlayer distances have a narrow distribution of values, very well defined system geometries are involved.

Work to date has revealed the following: the mass transport of polystyrene (nearly non-polar) entering into the layered silicate appears to be unhindered by the confinement of the chains and exhibits similar diffusion coefficient and temperature dependence to those of the polymer self-diffusion. The non-bulklike dependence of the intercalation diffusion coefficient on polymer-surface energetics and on polymer molecular weight is the focus of ongoing investigations, and will bring further insight into the nature and mechanisms of polymeric motions in ultra-confined geometries and near surfaces, in the presence of a large chemical potential gradient.

The local and global dynamic behavior of the polymer chains in the nanocomposites are markedly different from the bulk. Locally, intercalated PEO chains exhibit greater flexibility along the chain backbone as compared to those in the bulk. A coexistence of fast and slow relaxation modes for a wide range of temperatures, in conjunction with atomistic simulations of confined coils, mirrors the rich local dynamics behavior of these confined systems [74]. Intercalated PS and PEO chains display marked suppression - or absence - of cooperative dynamics such as exhibited at the glass transition. In addition, intercalated PEO chains do not display a melting transition, but behave similarly to amorphous chains in the melt even at temperatures significantly below the melting temperature of bulk PEO.

On a global scale, the linear viscoelastic behavior of the polymer chains in the nanocomposites, as detected by conventional rheometry, is dramatically altered when the chains are tethered to the surface of the silicate or are in close proximity to the silicate layers as in intercalated nanocomposites. Some of these systems show close analogies to other intrinsically anisotropic materials such as block copolymers and smectic liquid crystalline polymers and provide model systems to understand the dynamics of polymer brushes. Finally, the polymer meltbrushes exhibit intriguing non-linear viscoelastic behavior, which shows strainhardening with a characteric critical strain amplitude that is only a function of the interlayer distance. These results provide complementary information to that obtained for solution brushes using the SFA, and are attributed to chain stretching associated with the space-filling requirements of a melt brush.

\section{6}

\section{References}

1 (a) Giannelis EP, Mehrotra V, Tse OK, Vaia RA, Sung T (1992)in Synthesis and Processing of Ceramics: Scientific Issuesl, Rhine WE, Shaw MT, Gottshall RJ, Chen Y, eds MRS Proceedings: Pittsburgh, PA, 249: 547

(b )Wang MS, Pinnavaia TJ (1994) Chem Mater 6: 2216

(c) Aranda P, Ruiz-Hitzky E (1992) Chem Mater 4: 1395 
(d) Messersmith PB, Stupp SI (1992) J Mater Res 7: 2599

(e) Nazar LF et al. (1991) in Solid State Ionics II, MRS proceedings:Pittsburgh

(f) Lemmon JP, Lerner MM (1994) Chem Mater 6: 207

(g) Wu J, Lerner MM (1993) Chem Mater 5: 835

(h) Ding Y, Jones DJ, Maireles P, Roziere J (1995) Chem Mater 7: 562

(i) Mehrotra V, Giannelis EP (1990) in Polymer Based Molecular Composites, Shaefer DW, Mark JE, eds MRS Proceedings: Pittsburgh, PA, 171

Mehrotra V, Giannelis EP (1991) Solid State Commun 77: 155

Mehrotra V, Giannelis EP (1992) Solid State Ionics 51: 115

2 (a) Pinnavaia TJ (1983) Science 220: 365

(b) Theng BKG (1974) The Chemistry of Clay-Organic Reactions, John Wiley and Sons: NY

(c) Theng BKG (1979) Formation and Properties of Clay Polymer Complexes, Elsevier: NY 1979

(d )Solomon DH, Hawthorne DG (1991) Chemistry of Pigments and Fillers Krieger: Malabar, FL

(e) Whittingham MS, Jacobson AJ eds (1982) Intercalation Chemistry Academic Press: NY

3 (a) Cao G, Mallouk TEJ (1991) Solid State Chem 94: 59

(b) Pillion JE, Thompson ME (1991) Chem Mater :3 777

(c) Kanatzidis MG, Wu CG, Marcy HO, DeGroot DC, Kannewurf CR (1990) Chem Mater 2: 222

(d) Liu YJ, DeGroot DC, Schindler JL, Kannewurf CR, Kanatzidis MG (1991) Chem Mater 3: 992

(e) Kanatzidis MG, Wu CG, DeGroot DC, Schindler JL, Benz M, LeGoff E, Kannewurf CR (1993) in NATO ASI: Chemical Physics of Intercalation II, Fisher J eds Plenum Press

(f) Kato C, Kuroda K, Takahara H (1981) Clays and Clay Minerals 29: 294

(g) Ogawa M, Kuroda K, Kato C (1989) Clay Sci 7: 243

(h) Fukushima Y, Okada A, Kawasumi M, Kurauchi T, Kamigaito O (1988) Clay Min 23: 27

(i)Liu YJ, Schindler JL, DeGroot DC, Kannewurf CR, Hirpo W, Kanatzidis MG (1996) Chem Mater 8: 525

4 Lan T, Kaviratna PD, Pinnavaia TJ (1995) Chem Mater 7: 2144

5 Krishnamoorti RK, Vaia RA, Giannelis EP (1996) Chem Mater 8: 29

6 Giannelis EP (1996) Advanced Materials 8: 29

7 Vaia RA, Vasudevan S Krawiec W, Scanlon LG, Giannelis EP (1995) Adv Mater 7: 154

8 Vaia RA, Ishii H, Giannelis EP, (1993) Chem Mater 5: 1694;

Vaia RA (1995) Doctoral Thesis, Cornell University: Ithaca

9 Vaia RA, Teukolsky RK, Giannelis EP (1994) Chem Mater 6: 1017

10 Burnside SD, Giannelis EP (1995) Chem Mater 7: 1597

11 Vaia RA, Giannelis EP (1998) Macromolecules 30: 8000

12 Vaia RA, Jandt KD, Kramer EJ, Giannelis EP (1995) Macromolec 28: 8080; Vaia RA, Jandt KD, Kramer EJ, Giannelis EP (1996) Chem Mat 8: 2628

13 Wong S, Vasudevan S, Vaia RA, Giannelis EP, Zax D (1995) J Am Chem Soc 117: 7568; Wong S, Vasudevan S, Vaia RA, Giannelis EP, Zax D (1996) Solid State Ionics 86: 547

14 Vaia RA, Sauer BB, Tse OK, Giannelis EP (1997) J Polym Sci Part B: Polym Phys 35: 59

15 Krawiec W, Scanlon LG, Fellner JP, Vaia RA, Vasudevan S, Giannelis EP (1995) J Power Sources, 54: 310

16 Brindley SW (1980) Crystal Structure of Clay Minerals and their X-ray Diffraction, Brown G eds, Mineralogical Society: London; Güven N (1988) in Hydrous Phyllosilicates, Bailey SW eds, Reviews in Mineralogy Vol. 19, Mineralogical Society of America: Washington DC 497: 560

17 Messersmith PB, Giannelis EP (1994) Chem Mater 6: 1719

18 Messersmith PB, Giannelis EP (1995) J Polym Sci Part A: Polym Chem 33: 1047 
19 Usuki A, Kawasumi M, Kojima Y, Okada A, Kurauchi T, Kamigaito O (1993) J Mat Res 8: 1174; Usuki A, KojimaY, Kawasumi M, Okada A, Fukushima Y, Kurauchi T, Kamigaito O (1993) J Mat Res 8: 1179

20 Yano K, Usuki A, Karauchi T, Kamigaito O (1993) J Polym Sci, Part A: Polym Chem 31: 2493

21 Kojima Y, Usuki A, Kawasumi M, Okada A, Kurauchi T, Kamigaito O (1993) J Polym Sci Part A: Polym Chem 31: 983; Kojima Y, Usuki A, Kawasumi M, Okada A, Kurauchi T, Kamigaito O, Kaji K (1994) J Polym Sci Part B: Polym Phys 32: 625; ibid (1995) 33: 1039

22 de Gennes PG (1979) Scaling Concepts in Polymer Physics, Cornell University, Press: Ithaca, NY

23 Jinnai H, Smalley MV, Hasimoto T, Koizumi S (1996) Langmuir 12: 1199

24 Carrado KA, Thiyagarajan P, Elder DL (1996) Clays and Clay Minerals 44: 506

25 Muzny CD, Butler BD, Hanley HJM, Tsvetkov F, Peiffer DG (1996) Matt Lett 28: 379

26 Vaia RA (1995) Doctoral thesis, Cornell University, Ithaca, NY;

Vaia RA, Giannelis EP, Macromolecules 30: 7990

27 Scheutjens JMHM, and Fleer GJ., (1979) J. Phys. Chem 83 : 1619; (1980) J Phys Chem, 84: 178

28 Benshaul A, Szleifer I., Gelbart WM (1984) Proc. Nat. Acad. Sci., 81: 4601; Carignano MA, Szleifer I (1993) J. Chem. Phys., $98: 5001$

29 (a) Skipper NT, Sposito G, Chang FRC (1995) Clays and Clay Minerals 43: 285; ibid (1995) 43: 294

(b) Chang FRC, Skipper NT, Sposito G (1995) Langmuir 11: 2734

(c) Karaborni S, Smit B, Heidug W, Urai J, van Oort E (1996) Science 271: 1102

(d) Delville A, Sokolowski S (1993) J Phys Chemie 97: 6261

(e) Manias E, Chan YK, Giannelis EP, Panagiotopoulos AZ, in preparation

30 (a) Barrer RM, Craven RJB (1992) J Chem Soc, Faraday Trans 88: 645

(b) Breen C, Deane T, Flynn JJ, Reynolds D (1987) Clays and Clay Miner 17: 198

(c) Kärger J, Ruthven D (1992) Diffusion in Zeolites, J. Wiley \& Sons, NY

31 Green PF, Kramer EJ (1986) J Mater Res 1: 202; Antonietti M, Coutandin J, Grütter R, Sillescu RH (1984) Macromolecules 17: 798

32 Manias E, Genzer J, Chen H, Kramer EJ, Giannelis EP, submitted for publication

33 Manias E, Subbotin A, Hadziioannou G, ten Brinke G (1995) Molecular Physics 85: 101

34 Frank B, Gast AP, Russel TP, Brown HR, Hawker C (1985) Macromolecules 29: 6531

35 Green PF, Palmstrom CJ, Mayer JW, Kramer EJ (1985) Macromolecules 18: 501

36 Reiter GA, Demirel L, Granick S (1994) Science 263: 1741; Overney RM (1995) Trends in Polymer Science 3: 359; Pelletier E, Montfort JP, Lapique F (1994) J Rheol 38: 1151

37 Brik ME, Titman JJ, Bayle JP, Judeinstein P (1996) J Polym Sci Part B: Polym Phys 34: 2533

38 (a)Bitsanis IA, Pan C (1993) J Chem Phys 99: 5520; (b) Mansfield KF, Theodorou DN (1989) Macromolecules 22: 3143; (c) Manias E, Subbotin A, Hadziioannou G, ten Brinke G (1995) Molecular Physics 85: 1017; (d) Manias E (1995) Ph D thesis, University of Groningen, Groningen

39 Manias E, Hadziioannou G, ten Brinke G (1996) Langmuir 12: 4587

40 Toxvaerd S (1981) J Chem Phys 73: 1998; Magda JJ, Tirell M, Davis HT (1985) J Chem Phys 83: 1888; Bitsanis I, Magda JJ, Tirell M, Davis HAT (1987) J Chem Phys 87: 1733; Schoen M, Diestler DJ, Cushman JH (1987) J Chem Phys 87: 5464; Schoen M, Cushman J, Diestler D, Rhykerd C (1988) J Chem Phys 88: 1394; Schoen M, Rhykerd CJ, Diestler DJ (1989) Science 245: 1223; Bitsanis I, Somers S, Davis HT, Tirell M (1990) J Chem Phys 93: 3427; Thompson PA, Robbins MO (1990) Phys Rev A 41: 6830; Thompson PA, Robbins MO (1990) Science 250: 792; Diestler D, Schoen M, Hertzner A, Cushman J (1991) J Chem Phys 95: 5432; Lupkowski M, van Swol F (1991) J Chem Phys 95: 1995; Somers S, Davis TH (1992) J Chem Phys 96: 5389; Schoen M, Diestler DJ, Cushman JH (1994) J Chem Phys 100: 7707 
41 Mansfield KF, Theodorou DN (1989) Macromolecules 22: 3143; Thompson PA, Grest GS, Robbins MO (1992) Phys Rev Lett 68: 3448; Manias E, Hadziioannou G, Bitsanis I, ten Brinke G (1993) Europhysics Lett 24: 99; ibid (1996) 33: 371 Bitsanis I, Pan C (1993) J Chem Phys 99: 5520; Daivis P, Evans DJ (1994) J Chem Phys 100: 541; Koopman DC, Gupta S, Ballamudi RK, Westerman-Clark GB, Bitsanis I (1994) Chem Eng Sci 49: 2907; Manias E, Hadziioannou G, ten Brinke G (1994) J Chem Phys 101: 1721; Cieplak M, Smith ED, Robbins MO (1994) Science 265: 1209; Thompson PA, Grest GS, Robbins MO (1995) J Israel Chemie 35: 93

42 Bitsanis IA, ten Brinke GJ (1993) J Chem Phys 99: 3100

43 (a)Koopman DC, Gupta S, Ballamudi RK, Westerman-Clark GB, Bitsanis I (1994) Chem Eng Sci 49: 2907

(b) Ballamudi RK, Bitsanis IJ (1996) Chem Phys 105: 7774

44 Doi M, Edwards SF (1986) The theory of polymer dynamics $\$ 4.1 .2$ Oxford

45 In the case of intercalated polystyrene hybrids, the polystyrene was deintercalated and examined using DSC and GPC. The GPC traces before and after were identical, suggesting no degradation or cross-linking. Furthermore, the $\mathrm{T}_{\mathrm{g}}$ (a sensitive function of molecular weight in this range) for both pure and deintercalated PS was identical with superimposable DSC traces. On the other hand, PEO undergoes thermal degradation when heated above its melting temperature. Using FTIR we find that some degradation has taken place in the intercalated PEO samples. Since the techniques used to probe polymer dynamics (NMR, DSC and TSC) are very localized (smaller than 2-3 nm), the conclusions should be largely unaffected by the slight thermal degradation of PEO.

46 Frisch HL, Xue YP (1995) J Polym Sci Part A: Polym Chem 33: 1979

47 Keddie JL, Jones RAL, Cory RA (1994) Europhys Lett 27: 59; Keddie JL, Jones RAL, Cory RA (1994) Faraday Disc 98: 219

48 Wallace WE, van Zanten JH, Wu W, (1995) Phys Rev E 52: R3329; Forrest JA, DalnokiVeress K, Stevens RJ, Dutcher JR (1996) Phys Rev Lett 77: 2002; ibid 77: 4108

49 Mansfield KF, Theodorou DN, (1991) Macromolecules 24: 6283

50 Sauer BB, Hsiao BS (1993) J Poly Sci: Polym Phys 31: 917; Sauer BB, DiPaolo NV, Avakian P, Kampert WG, Starkweather HW (1993) J Poly Sci, Polym Phys 31: 1851; Sauer BB, Beckerbauer R, Wang L (1993) J Poly Sci: Polym Phys 31: 1861; Sauer BB, Avakian P (1992) Polymer 33: 5128

51 Cheng SZ, Wu ZQ, Wunderlich B (1987) Macromolecules 20: 2802; Cheng SZ, Cao MY, Wunderlich B (1986) Macromolecules 19: 1868

52 Krishnamoorti R, Giannelis EP; Unpublished data

53 Enikolopyan NS, Fridman ML, Stalnova IU, Popov VL (1990) Adv Polym Sci 96: 1

54 Krishnamoorti R, Giannelis EP (1997) Macromolecules , 30: 4097

55 Krishnamoorti R, Giannelis EP, Unpublished data

56 Rosedale JH, Bates FS (1990) Macromolecules 23: 2329; Koppi KA, Tirrell M, Bates FS, Almdal K, Colby RH (1993) J Phys II (Paris) 2: 1941

57 Larson RG, Winey KI, Patel SS, Watanabe H, Bruinsma R (1993) Rheologica Acta 32: 245; Patel SS, Larson RG, Winey KI, Watanabe H (1995) Macromolecules, 28: 4313

58 Kawasaki K, Onuki A (1990) Phys Rev 42: 3664

59 Rubinstein M, Obukhov SP (1993) Macromolecules 26: 1740

60 Halperin A, Tirrell M, Lodge TP (1992) Adv Polym Sci 100: 31

61 Ohta T, Enomoto Y, Harden JL, Doi M (1993) Macromolecules 26: 4928; Doi M, Harden JL, Ohta T (1993) Macromolecules 26: 4935

62 Witten TA, Leibler L, Pincus P (1990) Macromolecules 23: 824

63 Recently we have conducted rheological experiments wherein the end-tethered PCL nanocomposites were blended with pure PCL homopolymer. Rheological behavior, particularly the terminal zone slopes, obtained for $5 \%$ and $10 \%$ (obtained by blending equal weight fractions of PCL homopolymer with a 10 weight $\%$ PCL and 20 weight \% PCL respectively) were found to be similar to those obtained from the as-prepared nanocomposites. 
64 Watanabe H, Kuwahara S, Kotaka T (1984) J Rheol 28: 393; Adams JL, Graessley WW, Register RA (1994) Macromolecules 27: 6026

65 Agarwal S, Salovey R (1995) Polym Eng and Sci 35: 1241

66 Reiter G, Demirel AL, Granick S (1994) Science 263: 1741; Klein J, Perahia D, Warburg S (1991) Nature 352: 143; Granick S (1996) MRS Bulletin 21: 33; Cai LL, Peanasky J, Granick S (1996) Trends in Polym Sci 4: 47; Granick S, Demirel L, Cai L, Peanasky J (1995) Israel J of Chem 35: 75

67 Doyle PS, Shaqfeh ESG, Gast AP (1997) Phys Rev Lett 78: 1182

68 Fytas G, Anastasiadis SH, Seghrouchni R, Vlassopoulos D, Li JB, Factor BJ, Theobald W, Toprakcioglu C (1996) Science 274: 2041

69 Rabin Y, Alexander S (1990) Europhys Lett 13: 49; de Gennes PG (1979) Scaling Concepts in Polymer Physics, Cornell University Press: Ithaca, NY; Joanny JF (1992) Langmuir 8: 989; Subramanian G, Williams DRM, Pincus P (1996) Macromolecules 29: 4045; Milner ST (1991) Science 251: 905; Semenov AN (1995) Langmuir 11: 3560; BrochardWyart F (1993) Europhys Lett 23: 105; Brochard-Wyart F, Hervet H, Pincus P (1994) Europhys Lett 26: 511

70 Israelachvili JN, Tabor D (1972) Proc R Soc London A331: 19; Klein J (1983) J Chem Soc, Faraday Trans 1 79: 99; Overney RM (1995) Trends in Polym Sci 3: 359; Israelachvili JN (1992) Surf Sci Rep 14: 109

71 Klein J (1996) Ann Rev Mat Sci 26: 581

72 Szleifer I, Carignano MA (1996) Adv Chem Phys 94: 165

73 Krishnamoorti R, Giannelis EP (1998) Langmuir, in press

74 Hacket E, Manias E, Giannelis EP (1998) J Chem Phys, 108: 7410

Received: January 1998 